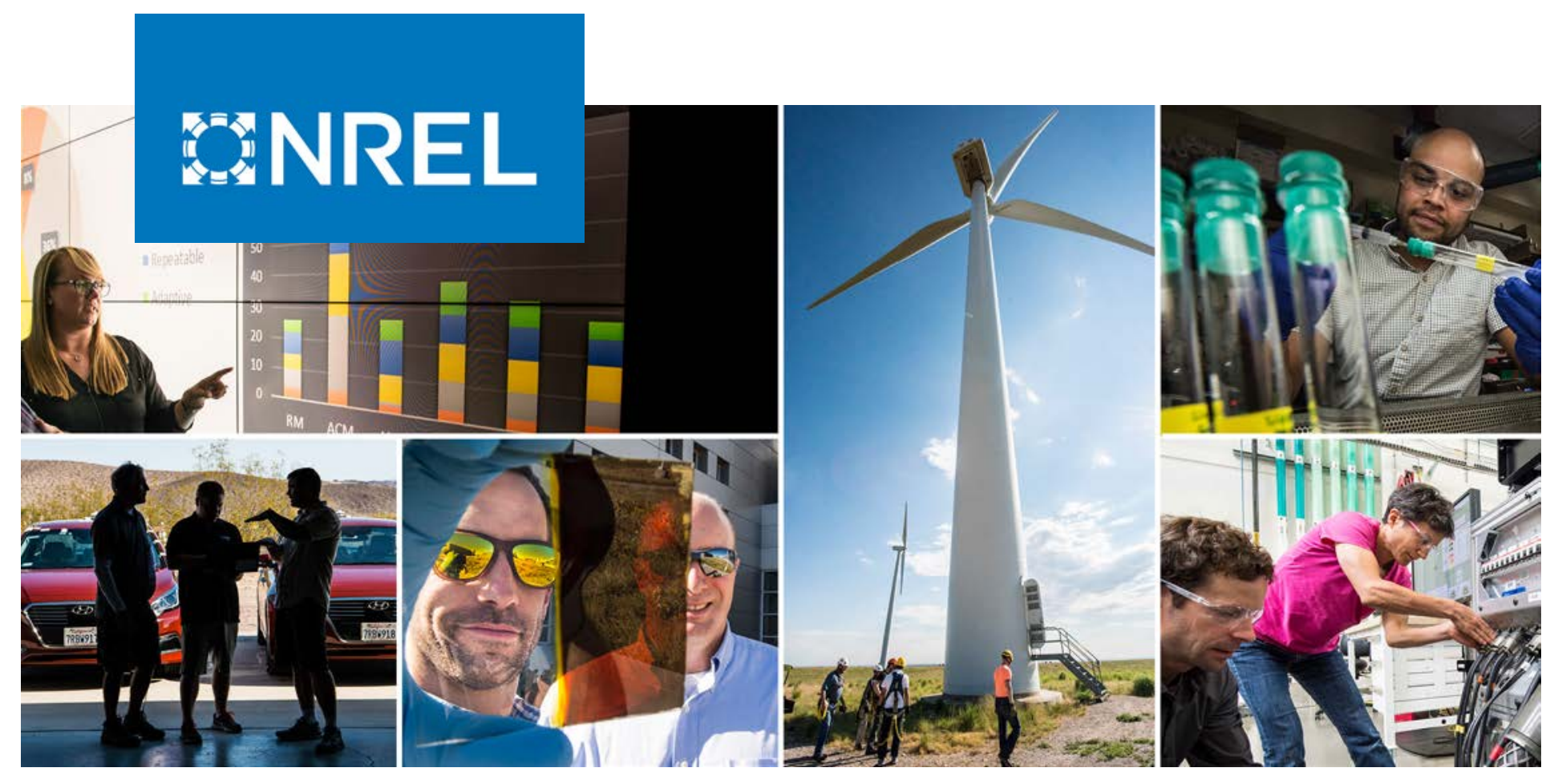

\title{
Providing Ramping Service with Wind to Enhance Power System Operational Flexibility
}

Xin Fang, ${ }^{1}$ Kwami Sedzro, ${ }^{1}$ Bri-Mathias Hodge, ${ }^{1}$ Jie Zhang, ${ }^{2}$ Binghui $\mathrm{Li}^{2}{ }^{2}$ and Mingjian $\mathrm{Cui}^{2}$

${ }^{1}$ National Renewable Energy Laboratory

${ }^{3}$ University of Texas at Dallas

NREL is a national laboratory of the U.S. Department of Energy Office of Energy Efficiency \& Renewable Energy

Operated by the Alliance for Sustainable Energy, LLC

This report is available at no cost from the National Renewable Energy Laboratory (NREL) at www.nrel.gov/publications.
Technical Report

NREL/TP-5D00-73643

March 2020 


\title{
GNREL
}

\section{Providing Ramping Service with Wind to Enhance Power System Operational Flexibility}

\author{
Xin Fang, ${ }^{1}$ Kwami Sedzro, ${ }^{1}$ Bri-Mathias Hodge, ${ }^{1}$ \\ Jie Zhang, ${ }^{2}$ Binghui $\mathrm{Li}^{2}{ }^{2}$ and Mingjian $\mathrm{Cui}^{2}$ \\ ${ }^{1}$ National Renewable Energy Laboratory \\ 2 University of Texas at Dallas
}

Suggested Citation

Fang, Xin, Kwami Sedzro, Bri-Mathias Hodge, Jie Zhang, Binghui Li, and Mingjian Cui. 2020.

Providing Ramping Service with Wind to Enhance Power System Operational Flexibility.

Golden, CO: National Renewable Energy Laboratory. NREL/TP-5D00-73643.

https://www.nrel.gov/docs/fy20osti/73643.pdf.

NREL is a national laboratory of the U.S. Department of Energy Office of Energy Efficiency \& Renewable Energy Operated by the Alliance for Sustainable Energy, LLC

This report is available at no cost from the National Renewable Energy Laboratory (NREL) at www.nrel.gov/publications.

Contract No. DE-AC36-08GO28308
Technical Report NREL/TP-5D00-73643 March 2020

National Renewable Energy Laboratory 15013 Denver West Parkway Golden, CO 80401 303-275-3000 • www.nrel.gov 


\section{NOTICE}

This work was authored in part by the National Renewable Energy Laboratory, operated by Alliance for Sustainable Energy, LLC, for the U.S. Department of Energy (DOE) under Contract No. DE-AC36-08GO28308. Funding provided by U.S. Department of Energy Office of Energy Efficiency and Renewable Energy Wind Energy Technologies Office. The views expressed herein do not necessarily represent the views of the DOE or the U.S. Government.

This report is available at no cost from the National Renewable Energy Laboratory (NREL) at www.nrel.gov/publications.

U.S. Department of Energy (DOE) reports produced after 1991 and a growing number of pre-1991 documents are available free via www.OSTI.gov.

Cover Photos by Dennis Schroeder: (clockwise, left to right) NREL 51934, NREL 45897, NREL 42160, NREL 45891, NREL 48097, NREL 46526.

NREL prints on paper that contains recycled content. 


\section{Foreword}

This project began in April 2016 and has a total duration of 3 years. It was conducted primarily at the National Renewable Energy Laboratory, in collaboration with the University of Texas at Dallas, and with advisory participation from the Electric Reliability Council of Texas. 


\section{Preface}

This project aims to develop an innovative, integrated, and transformative approach to mitigate the impact of wind ramping by providing flexible ramping products from wind power. The project will significantly contribute to the reduction of wind integration costs by making wind power dispatchable and allowing the efficient management of wind ramping characteristics. In addition, we collaborated with industry to test and validate the methodology, considering economic and reliability goals, by integrating the proposed methodology into the simulated market frameworks of two independent system operators: California Independent System Operator and the Electric Reliability Council of Texas. 


\section{Acknowledgments}

The authors thank Pengwei Du of the Electric Reliability Council of Texas and Qin Wang of the Electric Power Research Institute for their contributions to this project. This work was supported by the U.S. Department of Energy under Contract No. DE-AC36-08GO28308 with Alliance for Sustainable Energy, LLC, the Manager and Operator of the National Renewable Energy Laboratory. Funding provided by U.S. Department of Energy Office of Energy Efficiency and Renewable Energy Wind Energy Technologies Office. The views expressed in the article do not necessarily represent the views of the DOE or the U.S. Government. 


\section{List of Acronyms}

ACE

ARMA

ASV

CAISO

DA-SCED

FRP

GMM

ISOs

MISO

OpenSMEMS

OpSDA

PG\&E

PICP

PINC

RTD

RT-SCED

SCED

SCUC

SEM

TAMU

WIND Toolkit

WPRF average coverage error

autoregressive moving average model

average score value

California Independent System Operator

day-ahead security-constrained economic dispatch

flexible ramping products

Gaussian mixture model

independent system operators

Midcontinent Independent System Operator

Open-source Sequential Multi-timescale Electricity Market Simulation optimized swinging door algorithm

Pacific Gas and Electric Company

predictive interval coverage probability

predictive interval nominal confidence

real-time dispatch

real-time security-constrained economic dispatch

security-constrained economic dispatch

security-constrained unit commitment

synthetic evaluation metric

Texas A\&M University

Wind Integration National Dataset Toolkit

wind power ramp forecasting 


\section{Executive Summary}

Maintaining the power system balance requires controllable resources to adjust their power output to match the time-varying net load. This is becoming more challenging when the proportion of generation from variable and uncertain renewable resources in the system is high. It has been observed recently that the variability of the net load will bring two main negative impacts to power systems: (1) the ramp response of controllable resources will become taxed, and (2) the frequency of short-term generation scarcity events will increase as a result of shortages of ramping capacity.

In power system real-time operations, regulation services provide the only response option for balancing the variations in net load in the time frame of seconds or minutes. In the dispatch horizon, load-following resources are scheduled to provide the most economic solution to the expected level of variation. Currently, the expected level of variation can be met by residual capabilities of controllable resources. The challenge, however, is that system changes beyond the visibility of the dispatch horizon can leave the dispatchable resources with sufficient capacity but without ramp capability to respond when needed. This can lead to a short-term scarcity event.

This project developed an innovative, integrated, and transformative approach to mitigate the impact of net load ramping by providing flexible ramping products from wind power. The project facilitates the efficient management of wind ramping, leading to increased dispatchability and subsequent reduction in the cost of wind integration. In addition, the National Renewable Energy Laboratory team worked with industry to design the wind ramping product and will disseminate the findings related to system economics, flexibility, and market efficiency improvements. This project:

- Developed a probabilistic wind power ramp forecasting method to characterize and forecast ramps from a utility-scale perspective

- Analyzed and synthesized ramping products specific to the proposed test system(s)

- Designed flexible ramping products that can be implemented in a new market model to co-optimize energy, reserve, and ramping

- Validated benefits of incorporating wind ramp forecasts and improving wind power dispatch management and demonstrated potential economic and reliability benefits

- Developed an open-source Python-based market dispatch tool, the Open-source Sequential Multi-timescale Electricity Market Simulation (OpenSMEMS) toolbox and integrated the proposed ramping product model into it. The Pyomo package is used for the optimization modeling, and the Xpress solver is used for the MIP and LP optimization.

- Used OpenSMEMS to simulate an actual independent system operator large-scale system

- Created awareness of the benefits of flexible ramping products for the enhanced integration of wind energy by sharing methodologies and lessons learned with industry.

On the wind ramp forecast, the proposed method considers both the ramping features' dependence and wind power ramp forecasting error uncertainties by using the Gaussian mixture model marginal distribution. The model yields better results than conventional forecasting methods and lead to the lowest average coverage error, lowest average score value, and lowest synthetic evaluation metric. The proposed copula-based model (cp-WPRF) consistently presents 
the closest coverage probability curve and the smallest interval score regardless of the uncertainty distribution, demonstrating its robustness.

It has also been found that wind providing ramping services in day-ahead or hour-ahead markets can result in significant conventional generator dispatch displacement in real-time operation, as shown in Figure ES-1.

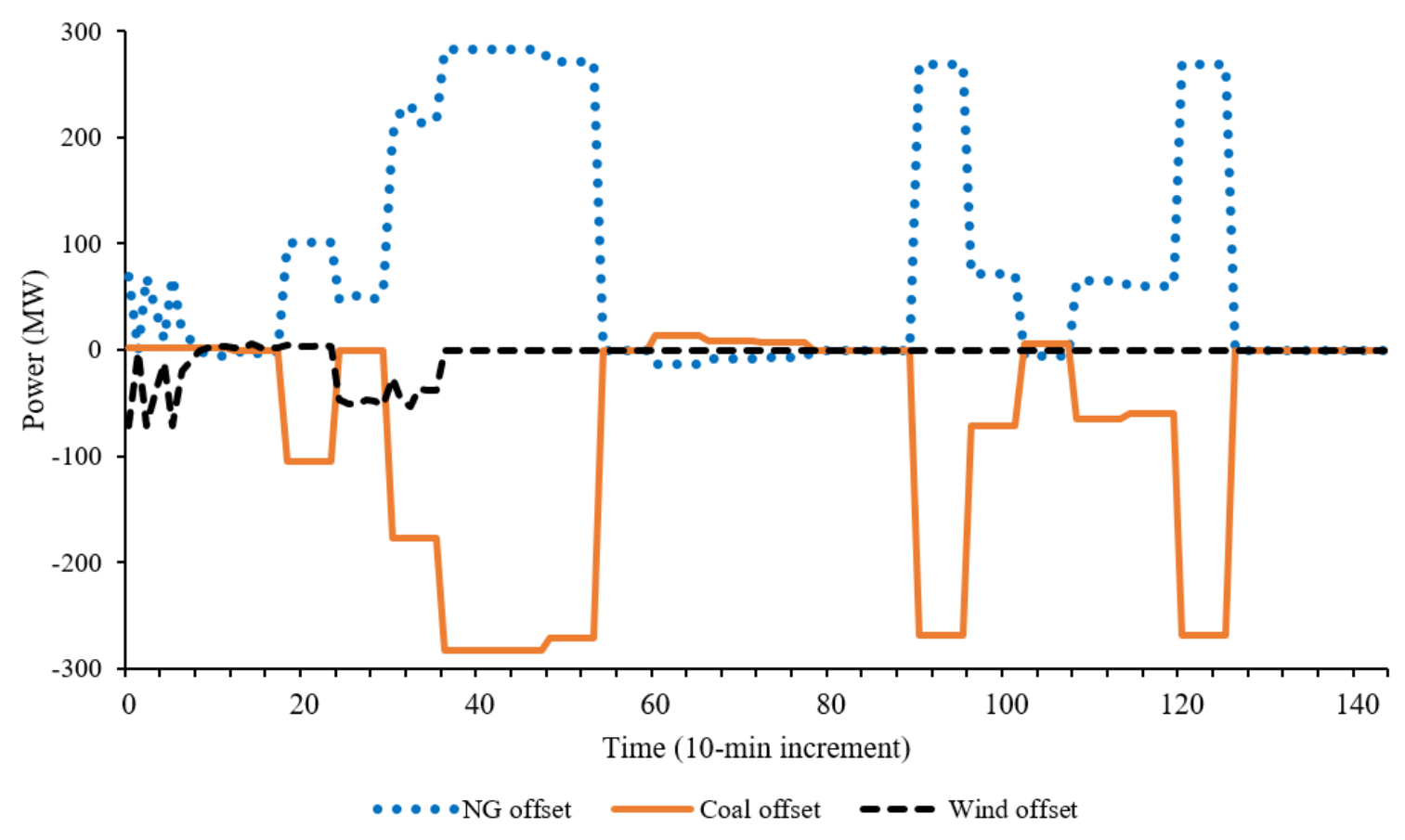

Figure ES-1. Change in real-time generation dispatch as a result of wind providing ramping services in the Texas A\&M University 2,000-bus system

This change in generation resource allocation translates into operational cost reduction. When provided in the day-ahead, wind ramping products result in a $72 \%$ ramp cost reduction and $6 \%$ total system cost reduction, whereas the real-time market wind ramping product provision leads to a $63 \%$ ramp cost reduction and $5 \%$ total system cost reduction for the 2000 -bus system considered. 


\section{Table of Contents}

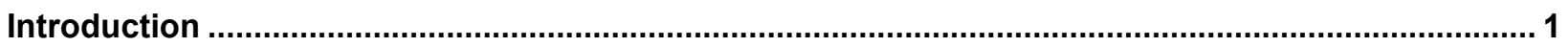

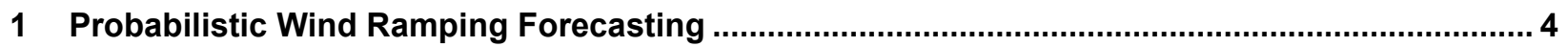

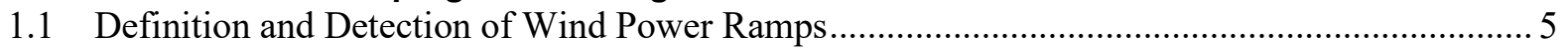

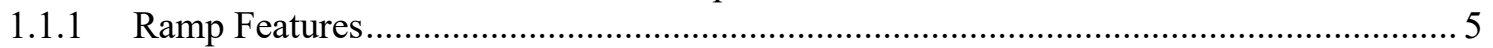

1.1.2 Uncertainty and Variability Characterization of Wind Power Ramp Forecasting ........... 5

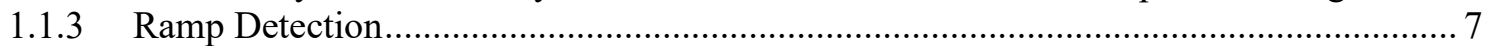

1.2 Conditional Probability Wind Power Ramp Forecast ............................................................ 8

1.2.1 Conditional Forecast of Uncertainty with Stochastic Dependence on Variability ........... 9

1.2.2 Multiple Conditions-Based cp-WPRF ................................................................... 9

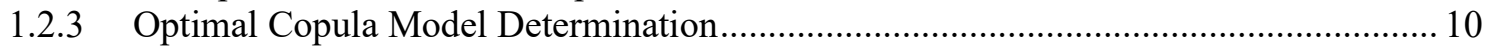

1.2.4 Development of cp-WPRF Model: An Example of Ramp Rate Forecasts .................... 10

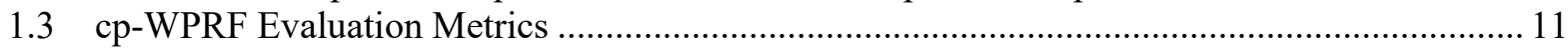

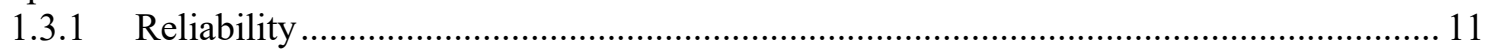

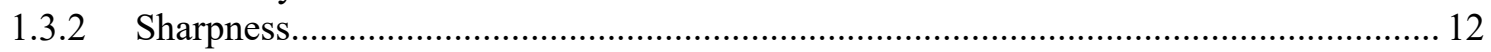

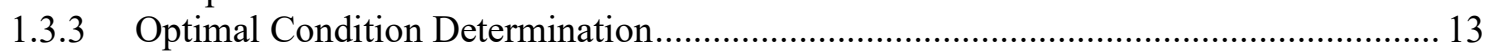

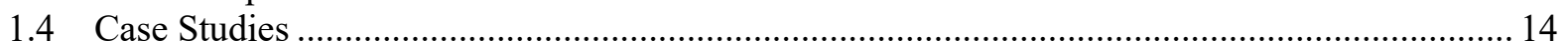

1.4.1 Comparisons of Different Probabilistic Wind Power Ramp Forecasting Models.......... 14

1.4.2 Robustness Analysis of cp-WPRF Models ............................................................... 19

2 Sequential Multi-Timescale Electricity Market Simulation Tool (OpenSMEMS) .......................... 22

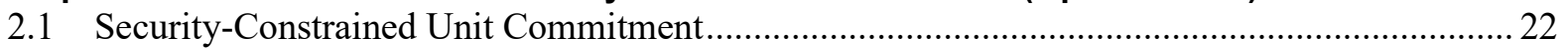

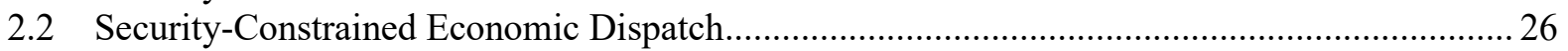

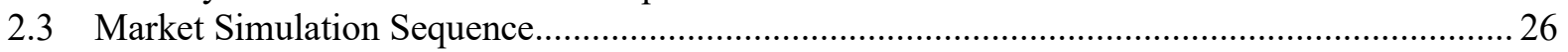

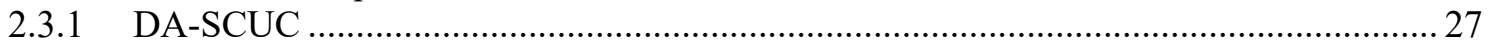

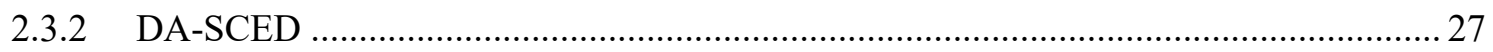

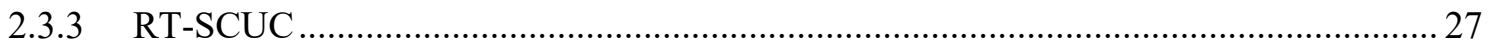

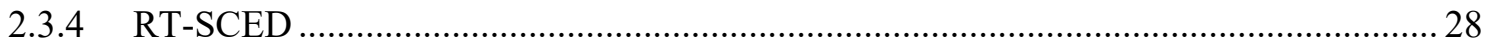

3 OpenSMEMS Use Case: Evaluating the Impact of Wind Providing Ramping Services ............... 30

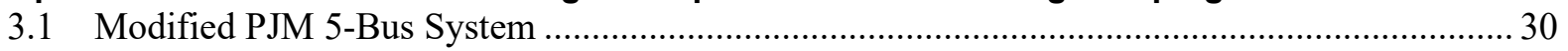

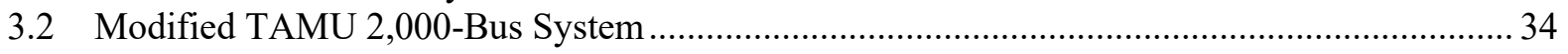

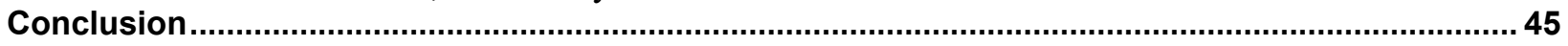

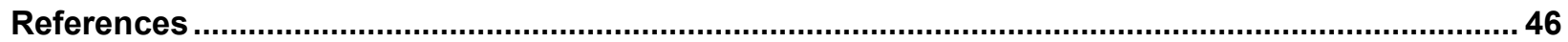




\section{List of Figures}

Figure ES-1. Change of real-time generation dispatch due to wind providing ramping services in the TAMU 2000-bus system.

Figure 2. A typical wind power ramp represented by different ramp features ....................................... 5

Figure 3. Deterministic forecasting results of ramp rate .................................................................. 6

Figure 4. Scatter plots of joint distributions for the ramp rate forecast error (y-axis) and two representative wind power ramp features ( $\boldsymbol{x}$-axis): ramp rate (a) and ramp duration (b).......6

Figure 5. Comparison of wind power ramp detection using the swinging door algorithm and OpSDA ...... 8

Figure 6. Overall framework of the developed cp-WPRF model: an example of ramp rate forecasts ....... 11

Figure 7. Flowchart of selecting the optimal condition .................................................................... 14

Figure 8. Comparison of different models of conditional probabilistic forecasts for ramp features in the Dallas area. From left to right: (a) magnitude, (b) duration, (c) start time, and (d) rate ........ 17

Figure 9. Comparison of different models of cp-WPRF for ramp rate in multiple regions. From left to right: (a) Miami, (b) Chicago, (c) New York City, and (d) Los Angeles............................... 18

Figure 10. Performance of different forecasting methods based cp-WPRF models (from left to right): ARMA-based cp-WPRF in (a), (b), (c); SEM values of multiple models in (d) .................... 20

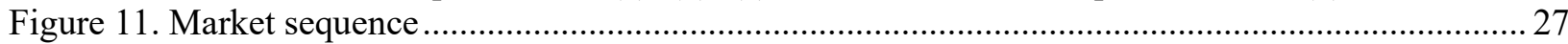

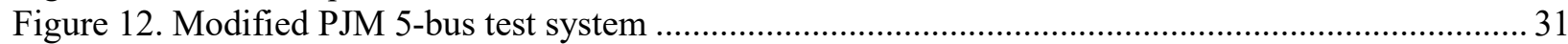

Figure 13. Demand and wind power forecasts at different market stages: PJM 5-bus ........................... 31

Figure 14. Dispatch by fuel type in different market segments in Sc.0 ............................................. 32

Figure 15. Impact of wind ramping product on system day-ahead ramp procurement cost at different wind

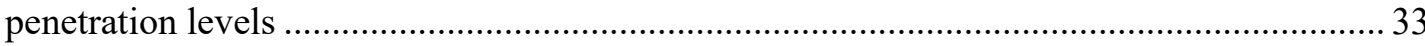

Figure 16. Impact of wind ramping product on system real-time ramp procurement cost at different wind

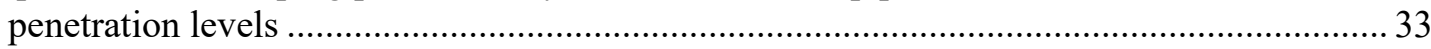

Figure 17. Day-ahead dispatch with and without WRP - 40\% wind penetration .................................... 34

Figure 18. Generation mix considered in the TAMU 2,000-bus base case ......................................... 35

Figure 19. Demand and wind power forecasts in different market segments: TAMU 2,000-bus system .. 36

Figure 20. Aggregate coal power output dispatch across all ramping service provision scenarios ............ 37

Figure 21. Aggregate natural gas power output dispatch across all ramping service provision scenarios . 38

Figure 22. Aggregate solar PV power output dispatch across all ramping service provision scenarios..... 39

Figure 23. Aggregate wind power output dispatch across all ramping service provision scenarios........... 40

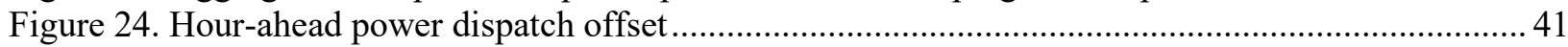

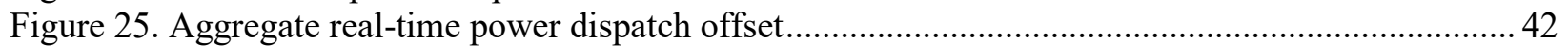

\section{List of Tables}

Table 1. Descriptions of Probabilistic Wind Power Ramp Forecasting Models...................................... 15

Table 2. Comparative Results for Different Ramp Features............................................................ 16

Table 3. Comparative Results for Ramp Rate in Multiple Regions....................................................... 19

Table 4. $\chi 2$ Statistics Comparison of GMM and Normal Distributions for Ramp Rate at Five Regions .. 19

Table 5. Optimal Copula Models and Best Conditions of Different Forecasting Methods-Based cp-WPRF

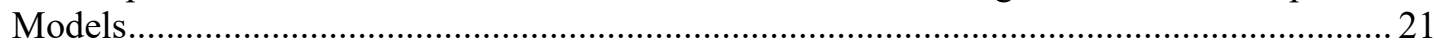

Table 6. Generation capacity shares in the original and the modified TAMU 2000-bus system .............. 35

Table 7. Sequential daily market cost summary ............................................................................... 43

Table 8. Simulation time summary of a sequential daily market operation.............................................. 43 


\section{Introduction}

This project aims to develop an innovative, integrated, and transformative approach to mitigate the impact of wind ramping by providing flexible ramping products from wind power. The project will significantly contribute to the reduction of wind integration costs by making wind power dispatchable and allowing the efficient management of wind ramping characteristics. In addition, we collaborated with industry to test and validate the methodology, taking into account economic and reliability goals, by integrating the proposed methodology into the simulated market frameworks of two independent system operators (ISOs): California Independent System Operator (CAISO) and the Electric Reliability Council of Texas (ERCOT).

Maintaining the power system balance requires controllable resources to adjust their power output to match the time-varying net load. This is becoming more challenging when the proportion of generation from variable and uncertain renewable resources in the system is high. It has been observed recently that the variability of the net load will bring two main negative impacts to power systems: (1) the ramp response of controllable resources will become taxed, and (2) the frequency of short-term generation scarcity events will increase as a result of shortages of ramping capacity.

In power system real-time operations, regulation services provide the only response option for balancing the variations in net load in the time frame of seconds or minutes. In the dispatch horizon, load-following resources are scheduled to provide the most economical solution to the expected level of variation. Currently, the expected level of variation can be met by residual capabilities of controllable resources. The challenge, however, is that the system changes beyond the visibility of the dispatch horizon can leave the dispatchable resources with sufficient capacity but without ramp capability to respond when needed. This can lead to a short-term scarcity event in the system.

This project developed an innovative, integrated, and transformative approach to mitigate the impact of net load ramping by providing flexible ramping products from wind power. The project facilitates the efficient management of wind ramping, leading to increased dispatchability and subsequent reduction in the cost of wind integration. In addition, the National Renewable Energy Laboratory team worked with industry to design the wind ramping product and will disseminate the findings related to system economics, flexibility, and market efficiency improvements. This project:

- Developed a probabilistic wind power ramp forecasting method to characterize and forecast ramps from a utility-scale perspective

- Analyzed and synthesized ramping products specific to the proposed test system(s)

- Designed flexible ramping products that can be implemented in a new market model to co-optimize energy, reserve, and ramping

- Validated benefits of incorporating wind ramp forecasts and improving wind power dispatch management and demonstrated potential economic and reliability benefits

- Developed an open-source market dispatch tool, the Open source Sequential Multitimescale Electricity Market Simulation (OpenSMEMS) toolbox, and integrated the proposed ramping product model into it 
- Used OpenSMEMS to simulate an actual ISO large-scale system

- Created awareness of the benefits of flexible ramping products for the enhanced integration of wind energy by sharing methodologies and lessons learned with industry.

To mitigate the uncertainty in real-time operations, several ISOs have initiated or launched ramping products in electricity market operation. The state-of-the-art ramping products in the electric power industry are described as follows.

- CAISO: "In August 2011, the California ISO Board of Governors approved the flexible ramping constraint interim compensation methodology. At that time the ISO committed to begin a stakeholder initiative to evaluate the creation of a flexible ramping product that will allow the ISO to procure sufficient ramping capability via economic bids. Through this initiative, the ISO will evaluate allocating costs to generation and load in accordance with cost causation principles. [CAISO, 2012]"

- Pacific Gas and Electric Company (PG\&E): "PG\&E supports the CAISO's efforts to identify through the FRP stakeholder process a market-based solution to the operational challenge of maintaining power balance in the Real-Time Dispatch (RTD) under increasing levels of variable energy resources (VERs). As these comments detail, however, key elements of the CAISO's proposed FRP market design changes remain unclear to PG\&E and other stakeholders. To achieve broad stakeholder support for the FRP initiative, the CAISO must explain much more precisely the mechanics of FRP procurement and settlement. Furthermore, PG\&E strongly encourages the CAISO to conduct robust market simulations as part of the FRP stakeholder process to demonstrate to stakeholders that the proposed market design changes are likely to yield reasonable market outcomes. [PG\&E, 2011]"

- Midcontinent Independent System Operator (MISO): "MISO recommends an approach that extends the concepts embedded in the Ramp-Up and Ramp-Down Headroom Capacity Constraints to improve the availability of ramp capability through new up ramp capability (URC) and down ramp capability (DRC) products. It is expected that the introduction of ramp capability products can provide an attractive approach to obtaining needed operational flexibility at a lower cost than other alternatives, providing both market and reliability benefits. Potential benefits include: reduced frequency of reserve shortages or transmission violations, less need to dispatch high cost resources, avoided cost of uneconomic $\mathrm{CT}$ commitments to provide ramp, reduced need for ad hoc operator actions such as RT adjustments in the UDS Offset MW and CT commitment providing increased consistency of market results, transparent pricing and incentives for the supply of ramp capability, reduced need for operator intervention in routine real-time market operations, freeing operator time to focus on other issues, etc. [MISO, 2013]"

Although several ISOs and utilities in the United States are realizing the importance of procuring adequate flexible resources in market operation and proposing new ramping products, they have not planned to use wind as a source of ramping service. Because many states have set up ambitious plans for the penetration level of wind power, we believe that the wind-friendly ramping product can provide an attractive approach to guarantee the needed operational flexibility and bring substantial economic benefits to the system.

The aim of the proposed wind-friendly flexible ramping product is to transform a negative characteristic of wind power ramping into an advantageous one. Through efficient management 
of wind ramps, wind power integration costs can be significantly reduced while simultaneously allowing the optimization of wind power as a ramping product in the market.

Main project initiatives are to:

1. Develop a probabilistic wind power ramp forecasting method to characterize and forecast ramps from a utility-scale perspective

2. Perform analysis and synthesis of ramping products specific to the proposed test system(s), allowing guidelines and recommendations to be derived with respect to spatiotemporal impacts and other case-specific considerations

3. Design flexible ramping products that can be implemented in a new market model to cooptimize energy, reserves, and ramping

4. Validate the benefits of incorporating wind ramp forecasts and improved management of wind power dispatch and demonstrate potential economic and reliability benefits

5. Continue to develop OpenSMEMS (formerly called GridLAB-ISO) and integrate the proposed ramping product model into it; use OpenSMEMS to simulate an ISO's system operations

6. Create awareness of the benefits of flexible ramping products for the enhanced integration of wind energy, sharing methodologies and lessons learned with industry. The National Renewable Energy Laboratory will work with utilities and the Electric Power Research Institute to share findings and methodologies with utilities and ISOs. 


\section{Probabilistic Wind Ramping Forecasting}

Wind power ramps are caused by large fluctuations in wind speed in a short time period and can threaten the secure and stable operation of power systems. Wind power ramp forecasting (WPRF), however, is still challenging for system operators, even though larger wind power penetrations are being seen in power systems worldwide, which makes wind power ramp forecasting significant for practical applications.

Wind power ramp forecasting methods can be divided into deterministic forecasts (d-WPRF) and probabilistic forecasts ( $\mathrm{p}-\mathrm{WPRF}$ ). The recent development of machine learning methods makes it possible to constitute deterministic wind power ramp forecasting models. The $\mathrm{p}$-WPRF is expected to provide more information on forecasting uncertainties of wind power ramps. Accurate information of $\mathrm{p}$-WPRF can improve the robustness of wind-friendly flexible ramping product design, thus achieving better cost-effectiveness of power market operations. Most existing $\mathrm{p}$-WPRF methods can be further categorized into two-step and one-step methods. The two-step p-WPRF method is realized by extracting all possible wind power ramps from a massive number of wind power scenarios, which are generated by using wind power forecasting techniques. The probabilistic characteristics of wind power ramp forecasting are then obtained through statistical analysis of the detected wind power ramps. Although employed in a wide range of studies, the two-step p-WPRF method is generally computationally expensive because of its dependence on a large number of wind power scenarios. In contrast, the one-step p-WPRF method directly forecasts ramp features based on historical measured wind power ramp characteristics and can avoid reliance on wind power scenario generation. Generally, wind power ramps can be characterized by four features: ramp rate, duration, magnitude, and start time. Most current literature, however, focuses on ramp rate forecasts while neglecting the stochastic dependence between different ramp features. To this end, a one-step, copula-based, conditional p-WPRF (cp-WPRF) model is developed using copula theory, which is able to model conditional probabilistic forecasts of wind power ramp features.

In this chapter, we seek to address two critical questions for balancing authorities with increasing penetrations of wind power in their power systems:

1. Is it possible to quantitatively evaluate the probabilistic information of wind power ramp features (e.g., rate, duration, magnitude, and start time)?

2. What is the impact of the stochastic dependence between WPRF uncertainties and different ramp features?

A cp-WPRF model is developed to characterize key ramp features: (1) using the Gaussian mixture model (GMM) to accurately fit the probability distributions of wind power ramp forecasting errors and ramp features, (2) using the copula theory to develop a cp-WPRF model to separately forecast each wind power ramp feature considering the stochastic dependence of ramping features, and (3) analyzing the probability information of conditional forecasts for ramp features. 


\subsection{Definition and Detection of Wind Power Ramps}

\subsubsection{Ramp Features}

A brief example of a wind power ramp is illustrated in Figure 1. As shown, one wind power ramp consists of four ramp features and one auxiliary variable. The four ramp features are ramp rate, duration, magnitude, and nonramp duration/start time, which are represented by symbols $R$, $D, M$, and $S$, respectively. The auxiliary variable is the start-time wind power output, represented by the symbol $P$. Note that the ramp start time can be calculated from the nonramp duration. Because ramp features are more practical for power system operations than the auxiliary variable, the developed wind power ramp forecasting model will forecast each ramp feature. Because four features and one auxiliary variable constitute one wind power ramp, the stochastic dependence among them needs to be modeled to forecast any ramp feature. To characterize the mutual dependence of wind power ramp features and the auxiliary variable, the copula theory is adopted for analytical analysis and introduced.

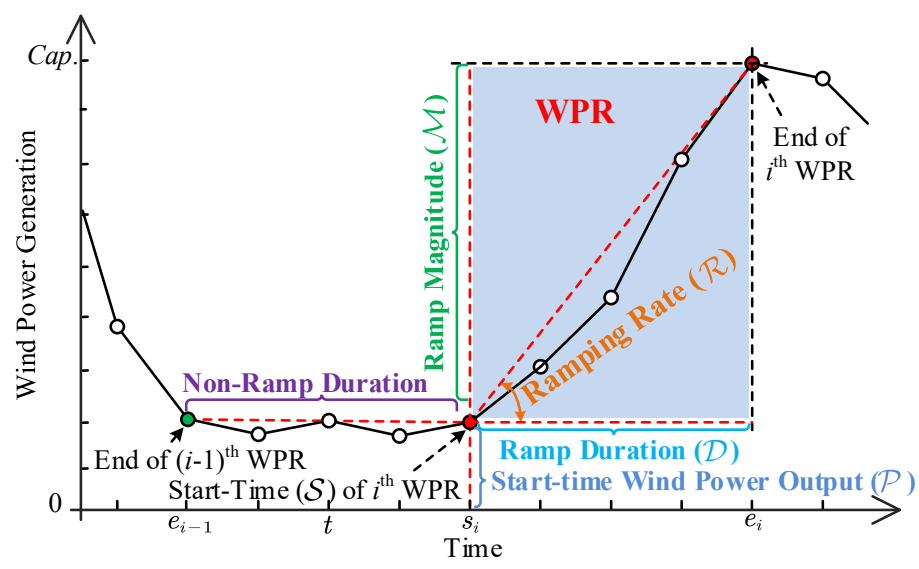

Figure 1. A typical wind power ramp represented by different ramp features

\subsubsection{Uncertainty and Variability Characterization of Wind Power Ramp Forecasting}

Similar to wind power, wind power ramp features present uncertainty and variability characteristics. Wind power ramp forecasting errors vary over time with different forecasting accuracies, which is a proxy for the uncertainty (see the green brace in Figure 2). The wind power ramp features change frequently with time, which is the variability (see the red brace in Figure 2). Taking the ramp rate forecast error as an example, Figure 3 shows the scatter plots of joint distributions of the ramp rate forecast error and two representative wind power ramp features: ramp rate and duration. Figure $3 \mathrm{a}$ shows that the ramp rate forecast error is directly proportional to the ramp rate. Figure $3 \mathrm{~b}$ shows that the ramp rate forecast error is inversely proportional to the ramp duration. This observation illustrates that the ramp rate forecast error correlates with wind power ramp features, such as ramp rate and duration; however, it is still challenging to model the stochastic dependence analytically when considering multivariate marginal distributions. Although a correlation coefficient can characterize the relationship between the ramp rate forecast error and ramp features, it cannot capture all the dependence information. Copulas are efficient at describing the correlations of stochastically dependent 
variables, and they have thus been adopted to model the probabilistic relationship between wind power ramp forecasting errors and wind power ramp features.

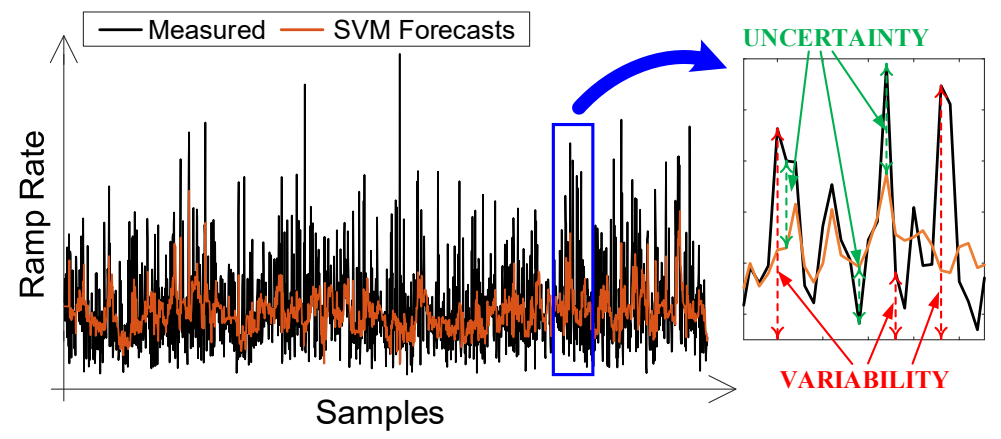

Figure 2. Deterministic forecasting results of ramp rate
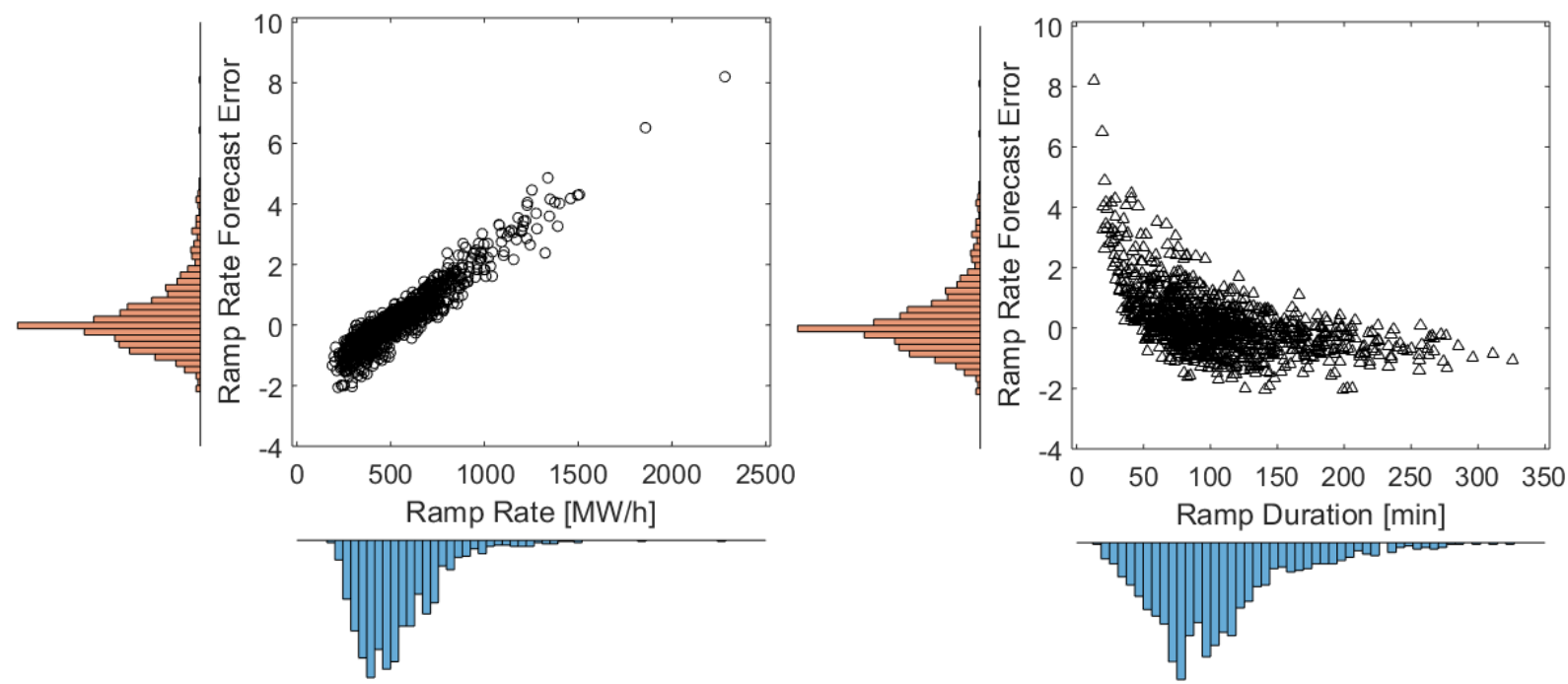

Figure 3. Scatter plots of joint distributions for the ramp rate forecast error ( $y$-axis) and two representative wind power ramp features ( $x$-axis): ramp rate (a) and ramp duration (b).

Currently, most of the literature uses unimodal distributions (normal distribution) or nonparametric distributions (kernel density estimation) to model the marginal distributions of the copula theory; however, unimodal distributions cannot accurately fit the irregular distributions of wind power ramp features, and nonparametric distributions cannot be solved analytically. To characterize the uncertainty and variability of wind power ramp features, the GMM distribution is used to accurately model the multimodal probability distributions of wind power ramp forecasting errors and wind power ramp features, respectively. The GMM distribution is formulated by:

$$
f\left(x_{r} \mid \boldsymbol{\Gamma}\right)=\sum_{i=1}^{N_{G}} \omega_{i} g_{i}\left(x_{r} \mid \mu_{i}, \sigma_{i}\right), r \in\{R, D, M, S\}
$$




$$
\int_{-\infty}^{\infty} f\left(x_{r} \mid \Gamma\right)=1 \Longrightarrow \sum_{i=1}^{N_{G}} \omega_{i} \int_{-\infty}^{\infty} g\left(x_{r} \mid \mu_{i}, \sigma_{i}\right)=1
$$

where $N_{G}$ is the total number of mixture components. $R$ is the wind power ramp features set, including the ramp rate $(R)$, ramp duration $(D)$, ramp magnitude $(M)$, and ramp start time $(S) . \Gamma$

defines the parameter set of all mixture components, i.e., $\Gamma=\left\{\omega_{i}, \mu_{i}, \sigma_{i}\right\}_{i=1}^{N_{G}} . \sigma$ is the standard deviation. $\mu$ is the mean value. $\omega$ is the weight. Each component $g\left(x_{r} \mid \mu, \sigma\right)$ conforms to a normal distribution, given by:

$$
g\left(x_{r} \mid \mu_{i}, \sigma_{i}\right)=\frac{1}{\sigma \sqrt{2 \pi}} \exp \left(-\frac{\left(x_{r}-\mu\right)^{2}}{2 \sigma^{2}}\right)
$$

where the integral of a normal distribution equals unity, i.e.:

$$
\sum_{i=1}^{N_{G}} \omega_{i}=1
$$

The GMM distribution has two unity attributes formulated in Eq. (2) and Eq. (4), which make it possible to use the expectation maximization algorithm to estimate all the parameters of mixture components. More detailed information about this algorithm can be found in [ Bodini, et al, 2017]. The uncertainty and variability of wind power ramp features are separately characterized by the GMM distribution with specific parameters.

\subsubsection{Ramp Detection}

The Wind Integration National Dataset (WIND) Toolkit [Draxl 2015] is used to construct the historical wind power ramps database, and the optimized swinging door algorithm (OpSDA) [ Zhu 2017] is used to automatically detect wind power ramps. In the OpSDA, the conventional swinging door algorithm with a predefined value is first applied to segregate the wind power data into multiple discrete segments. Then dynamic programming is used to merge adjacent segments with the same ramp direction and relatively high ramp rates. A brief description of the OpSDA is introduced in this section, and more details can be found in [Cui 2017]. Subintervals that satisfy the ramp rules are rewarded by a score function; otherwise, their score is set to zero. The current subinterval is retested as shown in subsection 1.1.2 after being combined with the next subinterval. This process is performed recursively until the end of the data set. A positive score function, $S_{c}$, is designed based on the length of the interval segregated by the swinging door algorithm. Given a time interval $(m, n)$ i n the forecasting horizon and an objective function, $S_{2}$, of the dynamic programming, a wind power ramp is detected by maximizing the objective function, $S_{2}$ :

$$
S_{2}(m, n)=\max _{m \leq v \leq n}\left[S_{c}(m, v)+S_{2}(v, n)\right], m<n
$$

s.t.

$$
S_{c}(m, n)>S_{c}(m, v)+S_{c}(v+1, n), \forall m<v<n
$$




$$
S_{c}(m, n)=(m-n)^{2} \times R L(m, n), \forall m<v<n
$$

where the positive score function, Sc, conforms to a superadditivity property in Eq. (6) and is formulated in Eq. (7). The ramp rule, $R L(m, n)$, is defined as the change in wind power magnitude without ramp duration limits. Thus, the wind power ramp is defined as the wind power change that exceeds the threshold (15\% of the installed wind capacity) without constraining the ramping duration. A brief example of wind power ramp detection in 1 day is illustrated in Figure 4. Figure 4a shows that the conventional swinging door algorithm detects only one wind power ramp without any optimization. As shown in Figure 4b, the OpSDA is able to combine the adjacent segments in the same direction and detect wind power ramps more accurately.
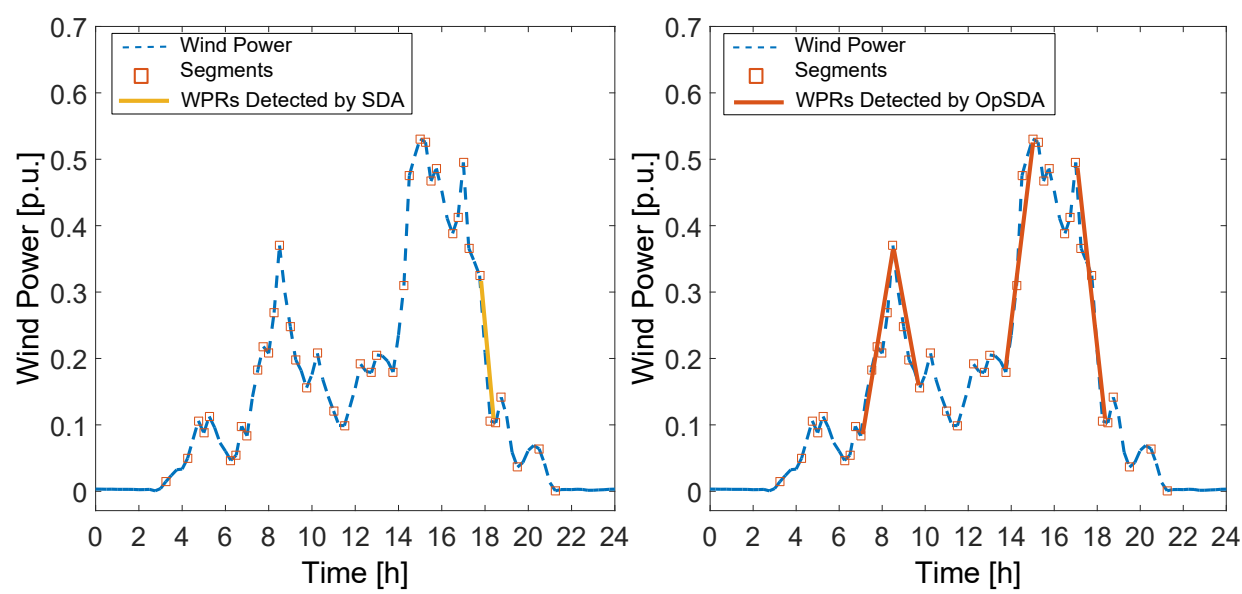

Figure 4. Comparison of wind power ramp detection using the swinging door algorithm and OpSDA

\subsection{Conditional Probability Wind Power Ramp Forecast}

Generally, the behavior of wind power ramp uncertainties is affected by wind power ramp variabilities, which is called stochastic dependence. In other words, the wind power ramp uncertainty and variability are stochastically dependent on each other. To analytically characterize the stochastic dependence between wind power ramp uncertainty and variability, the copula theory provides an effective way of capturing these correlations. Suppose that $x_{r}$ is the $r$ th uncertainty variable (wind power ramp forecasting errors), and $x_{r} \in\left\{x_{R} ; x_{D} ; x_{M} ; x_{S}\right)(r \in$ $R) ; y_{c}$ is the $c$ th random variability (or condition) variable (wind power ramp features and auxiliary variable), and $y_{c} \in\left\{y_{R} ; y_{D} ; y_{M} ; y_{S} ; y_{P}\right\}(c \in R \cup\{P\})$. The joint cumulative distribution function $F_{X_{r} Y_{C}}$ represents the stochastic dependence, given by:

$$
F_{X_{r} Y_{c}}\left(x_{r}, y_{c}\right)=F_{C}\left(F_{X_{r}}\left(x_{r}\right), F_{Y_{c}}\left(y_{c}\right)\right)
$$

where $F_{X_{r}}$ and $F_{Y_{C}}$ are the marginal cumulative distribution functions of wind power ramp uncertainty and variability that transform $x_{r}$ and $y_{c}$ into the uniform distributions, respectively. $F_{C}(\cdot)$ is the copula cumulative distribution function. In this way, the copula theory transforms the stochastic dependence problem into modeling $F_{X_{r}}, F_{Y_{C}}$, and $F_{C}(\cdot)$. 


\subsubsection{Conditional Forecast of Uncertainty with Stochastic Dependence on Variability}

Because of the stochastic nature of wind power ramps, a change in random wind power ramp forecasting errors would occur when the condition variables are altered, which is regarded as the stochastic dependence of wind power ramp uncertainty on variability. The joint distribution of wind power ramp forecasting errors and the conditional variable can be modeled by the copula function. The joint probability density function $f_{X_{r} Y_{c}}\left(x_{r} ; y_{c}\right)$ is formulated with the marginal probability density functions of $x_{r}, y_{c}$, and the copula probability density function $f_{C}(\cdot)$. Given that the point forecast of a single conditional variable is $y_{c}=\hat{R}_{c}$, the conditional probability density function of wind power ramp forecasting errors can be expressed as:

$$
\begin{aligned}
& f_{X_{r} \mid Y_{c}}\left(x_{r} \mid \hat{R}_{c}\right)=\frac{f_{X_{r} Y_{c}}\left(x_{r}, \hat{R}_{c}\right)}{f_{Y_{c}}\left(\hat{R}_{c}\right)} \\
= & f_{c}\left(F_{X_{r}}\left(x_{r}\right), F_{Y_{c}}\left(\hat{R}_{c}\right)\right) \cdot f_{X_{r}}\left(x_{r}\right)
\end{aligned}
$$

where the wind power ramp uncertainty variable $\left(x_{r}\right)$ pool $x_{r} \in\left\{x_{R} ; x_{D} ; x_{M} ; x_{S}\right)$ includes the wind power ramp uncertainties of four wind power ramp features. The dependent condition $\left(y_{c}\right)$ pool in $y_{C} \in\left\{y_{R} ; y_{D} ; y_{M} ; y_{S} ; y_{P}\right\}$ includes all the possible variables that are correlated with the wind power ramp uncertainty. Here, the dependent conditional variable pool consists of four wind power ramp features and start-time wind power output $(P)$. Note that the aforementioned pool definitions can be extended by balancing authorities for further studies. Each uncertainty or condition variable is normalized by:

$$
x=\frac{x_{\text {means. }}-\mu}{\sigma}
$$

where $\mu$ and $\sigma$ represent the mean value and standard deviation of uncertainty or condition variables, respectively.

\subsubsection{Multiple Conditions-Based cp-WPRF}

Copula theory can also be used to establish the multiple-conditions based cp-WPRF model by expanding Eq. (9). Given that point forecasts of multiple conditional variables are $y_{1}=\hat{R}_{1}, y_{2}=$ $\hat{R}_{2}, \cdots, y_{c}=\hat{R}_{c}$, the conditional probability density function of wind power ramp forecasting errors, namely $f_{X_{r} \mid Y_{1} Y_{2} \cdots Y_{c}}\left(x_{r} ; \hat{R}_{1}, \hat{R}_{2}, \cdots, \hat{R}_{c}\right)$, can be expressed. Unlike the single condition-based cp-WPRF, multiple conditional variables $\left(y_{1} ; y_{2} ; \cdots ; y_{c}\right)$ are selected from the dependent condition pool in Eq. (11) based on the constraint in Eq. (12). By varying the $c$ value, all the possible conditions can be considered.

$$
\begin{gathered}
y_{1}, y_{2}, \cdots, y_{c} \in\left\{y_{R} ; y_{D} ; y_{M} ; y_{S} ; y_{P}\right\} ; c \in R \cup\{P\} \\
y_{1} \neq y_{2} \neq \cdots \neq y_{c}
\end{gathered}
$$

Overall, the conditional distribution of wind power ramp uncertainty variables consists of the copula-based conditional probability density function as the variant multiplier and the marginal GMM distribution as the base. 


\subsubsection{Optimal Copula Model Determination}

To choose the optimal copula model, the Bayesian information criterion is used to assess the performance of different copula models. Thus, the optimal copula model is chosen by minimizing the Bayesian information criterion, formulated by:

$$
\arg \min N_{P} \ln N_{S}-2 \ln \left[\sum_{t=1}^{N_{S}} \ln f_{C}\left(u_{t}, v_{1, t}, \cdots, v_{c, t} ; \hat{\theta}\right)\right]
$$

where $N_{P}$ is the number of parameters in a copula model. $N_{S}$ is the number of measured samples. For the Gaussian copula, $N_{P}=c(c+1) / 2$. For the $t$ copula, $N_{P}=1+c(c+1) / 2$. For the Archimedean copula family, $N_{P}=1$.

The optimal copula model is determined by the Bayesian information criterion. In statistics, the Bayesian information criterion is a criterion used for model selection among a finite set of models. The copula model with the minimum Bayesian information criterion is preferred. The minimum Bayesian information criterion is calculated using cumulative distribution functions that transform the ramping feature data into uniform distributions. Different cumulative distribution function profiles of each ramping feature might generate a different optimal copula model.

\subsubsection{Development of cp-WPRF Model: An Example of Ramp Rate Forecasts}

Based on the optimal copula model, the predictive intervals of wind power ramp uncertainties $\left[x_{r, t}^{\alpha_{L}}, x_{r, t}^{\alpha_{U}}\right]$ can be calculated by using the conditional probability density function in Eq. (9). A predictive interval $\left(I_{r, t}^{\beta}\right)$ of the forecasted wind power ramps with a nominal coverage rate (1$\beta$ ) can be expressed with the lower bound, $\hat{R}_{r, t}^{\alpha_{L}}$, and the upper bound, $\hat{R}_{r, t}^{\alpha_{H}}$, given by:

$$
I_{r, t}^{\beta}=\left[\hat{R}_{r, t}^{\alpha_{L}}, \hat{R}_{r, t}^{\alpha_{U}}\right]=\hat{R}_{r, t}^{S V M}+\left[x_{r, t}^{\alpha_{L}}, x_{r, t}^{\alpha_{U}}\right]
$$

where the lower and upper nominal proportions $\alpha_{L}$ and $\alpha_{U}$ equal $\beta / 2$ and $(1-\beta / 2)$, respectively. The inverse function of the conditional cumulative distribution function, however, cannot be analytically deduced. Alternatively, we use the Newton-Raphson method to obtain the numerical solution. Taking the ramp rate forecasts as an example, the lower bound of the ramp rate uncertainty is generated based on the copula probability density function and the lower nominal proportion $\alpha_{L}$. The overall framework for generating the cp-WPRF of the ramp rate is illustrated in Figure 5, which mainly consists of four major steps: deterministic ramp rate forecast, marginal distribution fit, optimal copula model selection, and determining the best conditions-based cp-WPRF model. The four major steps are described as follows:

- Step 1: Based on the measured wind power ramp data of ramp features, a machine learning method (i.e., support vector machine) is used to separately generate deterministic forecasts of all wind power ramp features and the corresponding wind power ramp forecasting errors.

- Step 2: Each wind power ramp feature and its forecasting errors are characterized by the GMM distribution, which is used as the marginal distribution in copula models. 
- Step 3: Parameters of the copula models are estimated by the ML/CML method. The best copula model is chosen based on the minimum Bayesian information criterion.

- Step 4: The predictive intervals of ramp rate $I_{r, t}^{\beta}$ are calculated as the combination of the deterministic forecasts and the wind power ramp uncertainties predictive intervals (see Eq. (14)). The best conditions-based cp-WPRF model is determined by the quality of the predictive intervals with evaluation metrics.

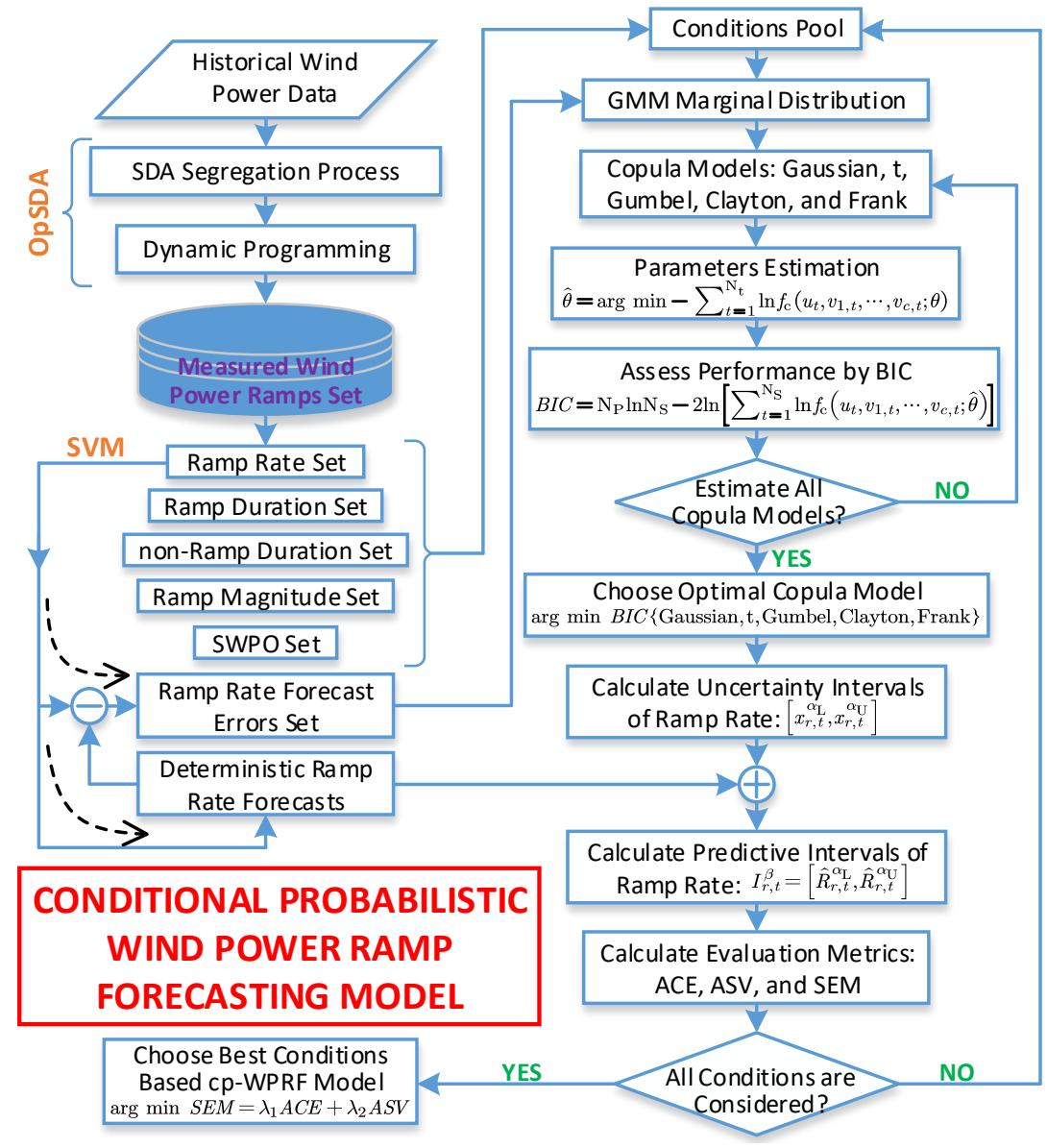

Figure 5. Overall framework of the developed cp-WPRF model: an example of ramp rate forecasts

\section{$1.3 \mathrm{cp}-W P R F$ Evaluation Metrics}

To evaluate the performance of cp-WPRF at different conditions, two predictive interval-based metrics - reliability and sharpness - are adopted and briefly introduced in this section. Reliability indicates the correct degree of a cp-WPRF assessed by the hit percentage. Sharpness indicates the uncertainty conveyed by the cp-WPRF.

\subsubsection{Reliability}

The forecasted wind power ramp features are expected to lie within the predictive interval bounds with a prescribed probability termed the nominal proportion. It is expected that the coverage probability of obtained predictive intervals will asymptotically reach the nominal level of confidence (ideal case) during the full wind power ramps. Predictive interval coverage probability (PICP) is a critical measure for the reliability of the wind power ramp predictive 
intervals, formulated as $P I C P=\frac{1}{N_{t}} \sum_{t=1}^{N_{t}} \phi_{t}^{\beta} \times 100 \%$, where the indicator of predictive interval coverage probability $\left(\phi_{t}^{\beta}\right)$ is defined in Eq. (15). Theoretically, the predictive interval coverage probability should be close to the corresponding predictive interval nominal confidence (PINC). The average coverage error (ACE) metric formulated in Eq. (16) should be as close to zero as possible. A smaller absolute ACE indicates more reliable predictive intervals of wind power ramps.

$$
\begin{gathered}
\phi_{t}^{\beta}=\left\{\begin{array}{l}
1, \hat{R}_{r, t} \in I_{r, t}^{\beta} \\
0, \hat{R}_{r, t} \notin I_{r, t}^{\beta}
\end{array}\right. \\
A C E=\frac{1}{N_{S L}} \sum_{j=1}^{N_{S L}}\left|\frac{1}{N_{t}} \sum_{t=1}^{N_{t}} \phi_{t}^{\beta_{j}}-P_{I N C^{\beta}}\right| \times 100 \%
\end{gathered}
$$

where $N_{S L}$ is the number of significance levels. $N_{t}$ is the number of test samples.

\subsubsection{Sharpness}

Sharpness is related to the interval size of different significance levels. The mean size of the predictive intervals $\left(\delta_{r}^{\beta}\right)$ at nominal coverage rate $(1-\beta)$ is:

$$
\delta_{r, t}^{\beta}=\frac{1}{N_{t}} \sum_{t=1}^{N_{t}}\left(x_{r, t}^{\alpha_{U}}-x_{r, t}^{\alpha_{L}}\right) \times 100 \%
$$

The interval score $S c_{r}^{\beta}\left(\hat{R}_{r, t}\right)$ rewards narrow predictive intervals and assesses a penalty if a target does not lie within estimated predictive intervals.

$$
S c_{r}^{\beta}\left(\hat{R}_{r, t}\right)=\left\{\begin{array}{c}
2 \beta \delta_{r}^{\beta}\left(\hat{R}_{r, t}\right)+r\left(\hat{R}_{r, t}^{\alpha_{L}}-\hat{R}_{r, t}\right), \text { if } \hat{R}_{r, t}<\hat{R}_{r, t}^{\alpha_{L}} \\
2 \beta \delta_{r}^{\beta}\left(\hat{R}_{r, t}\right), \text { if } \hat{R}_{r, t} \in I_{r, t}^{\beta} \\
2 \beta \delta_{r}^{\beta}\left(\hat{R}_{r, t}\right)+r\left(\hat{R}_{r, t}-\hat{R}_{r, t}^{\alpha_{U}}\right), \text { if } \hat{R}_{r, t}>\hat{R}_{r, t}^{\alpha_{L}}
\end{array}\right.
$$

The average score value (ASV) can be employed to comprehensively evaluate the overall skill of wind power ramp predictive intervals to assess the sharpness, given by:

$$
A S V=\frac{1}{N_{S L} N_{t}} \sum_{j=1}^{N_{S L}} \sum_{t=1}^{N_{t}} S c_{r}^{\beta}\left(\hat{R}_{r, t}\right) \times 100 \%
$$

Generally, smaller ACE and ASV values indicate better forecasting performance. To examine the trade-off between the reliability and sharpness metrics, the synthetic evaluation metric (SEM) is formulated by:

$$
S E M=\lambda_{1} \overline{A C E}+\lambda_{2} \overline{A S V}
$$


where ACE and ASV are normalized by Eq. (10). $\lambda_{1}$ and $\lambda_{2}$ are the weight coefficients (here, $\lambda_{1}=\lambda_{2}=0.5$ ). The weights could be adjusted based on the balancing authorities' (or other stakeholders') preferences between the two metrics.

\subsubsection{Optimal Condition Determination}

To select the optimal condition from the conditions pool, the objective function is constructed by minimizing the SEM metric for each correlated condition, given by:

$$
\underset{i \in \Omega}{\arg \min } S E M_{i}=\lambda_{1, i}{\overline{A C E_{i}}}+\lambda_{2, i} \overline{A S V}_{i}
$$

where $\Omega$ is the set of the conditions pool.

Figure 6 shows the procedure of selecting the optimal condition from the conditions pool, which is described as follows:

- Step 1: Prepare a conditions pool and choose the $i$ th condition from the conditions pool.

- Step 2: select the optimal copula model, calculate the predictive intervals, and calculate the evaluation metrics $S E M_{i}$ in Eq. (21).

- Step 3: If $i=1$, the optimal SEM is set as $S E M_{o p t, 1}=S E M_{1}$; otherwise, compare the ith SEM $S E M_{i}$ with the optimal SEM $S E M_{o p t, 1}$ in the last $(i-1)$ th iteration.

- Step 4: If $S E M_{i}<S E M_{o p t, i-1}$, the optimal SEM is replaced by $S E M_{o p t, i} \leftarrow S E M_{i}$ and $i_{\text {opt }} \leftarrow i$; otherwise, this step is skipped, and go to Step 5 .

- Step 5: Evaluate the termination condition. If the condition index $i$ is smaller than the total number of conditions $i<$ length $(\Omega)$, the index is updated by $\mathrm{i}=i+1$, and it returns to Step 1; otherwise, the iteration calculation is terminated. The optimal condition is selected as the $i_{\text {opt }}$ th condition. 


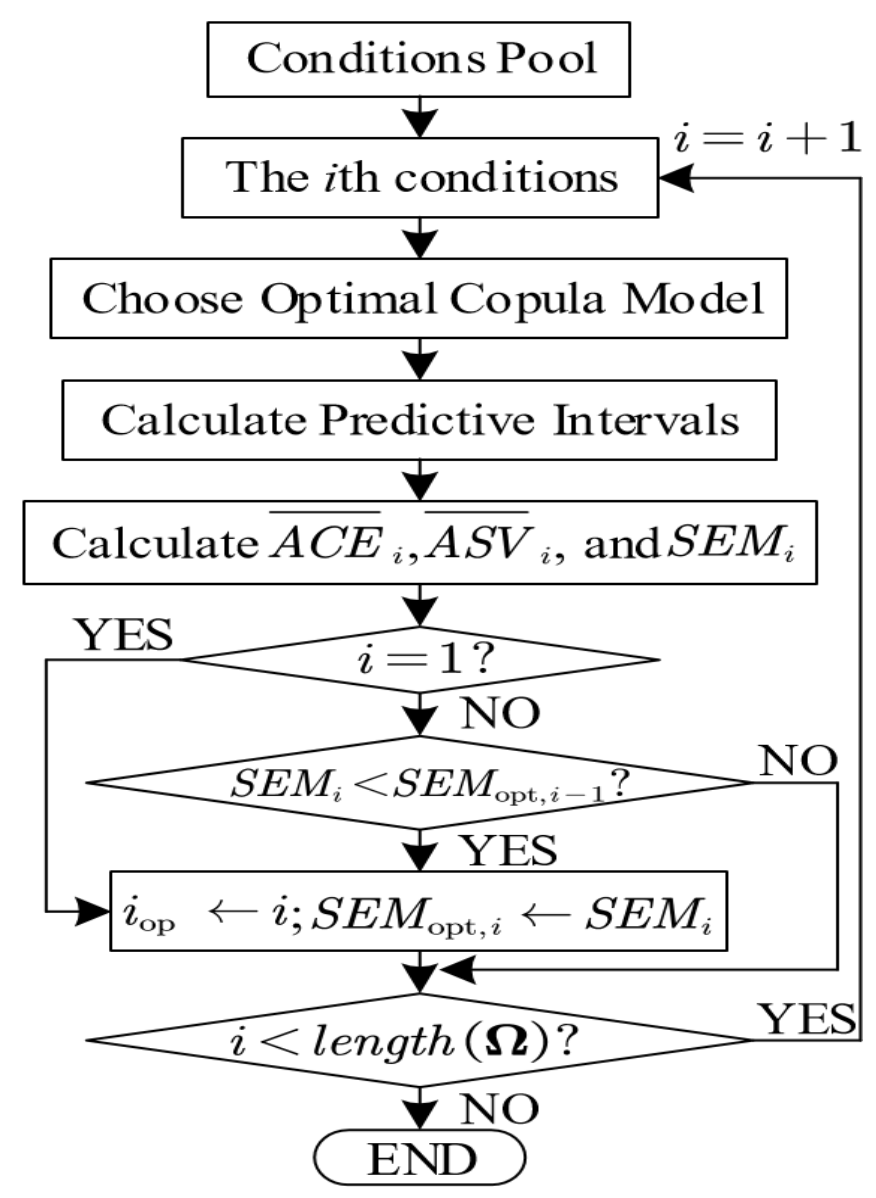

Figure 6. Flowchart of selecting the optimal condition

\subsection{Case Studies}

The developed cp-WPRF model is evaluated using data from the WIND Toolkit. The data represents wind power generation from January 1, 2007, to December 31, 2012. The wind power plants used in this analysis are located in the regions of Dallas, Miami, Chicago, Los Angeles, and New York with a 5-minute data resolution. The total rated wind power capacities are 10,028 MW, 9,555 MW, 9,974 MW, 10,119 MW, and 9,825 MW, respectively. There are approximately $1,585,1,121,1,819,1,245$, and 1,080 wind power ramps in each location, respectively. The last 140 wind power ramps are used for testing. The remaining wind power ramps are used for training. The door width of the OpSDA is set as $0.2 \%$ of the rated capacity.

\subsubsection{Comparisons of Different Probabilistic Wind Power Ramp Forecasting Models}

To verify the effectiveness of the developed cp-WPRF model, four probabilistic wind power ramp forecasting models are used for comparisons. The detailed information on the four models is described as follows and also summarized in Table 1.

- Model 1: considers only the ramp features' dependence without modeling wind power ramp forecasting error uncertainties 
- Model 2: considers only the wind power ramp forecasting error uncertainties without modeling the ramp features' dependence

- Model 3: considers both the ramp features' dependence and wind power ramp forecasting error uncertainties by using the normal marginal distribution

- Model 4 (proposed): considers both the ramping features' dependence and wind power ramp forecasting error uncertainties by using the GMM marginal distribution.

Two cases are studied to compare the performance of four probabilistic wind power ramp forecasting models: (1) Case 1: wind power ramp forecasting results of different ramp features in the same region and (2) Case 2: wind power ramp forecasting results of the same ramp feature in different regions.

Table 1. Descriptions of Probabilistic Wind Power Ramp Forecasting Models

\begin{tabular}{lclc}
\hline Wind Power Ramp & \multicolumn{3}{l}{ Descriptions } \\
\cline { 2 - 4 } Forecasting Models & Marginal Distribution & $\begin{array}{l}\text { Wind Power Ramp } \\
\text { Forecasting Error } \\
\text { Uncertainty }\end{array}$ & Copula Dependence \\
\hline Model 1 & GMM & - & $\checkmark$ \\
Model 2 & GMM & $\checkmark$ & - \\
Model 3 & Normal & $\checkmark$ & $\checkmark$ \\
Model 4 (Proposed) & GMM & $\checkmark$ & $\checkmark$ \\
\hline
\end{tabular}

Case 1: The wind power ramp data of the Dallas area are used as an extensive study in this case, i.e., different ramp features with multiple wind power ramp forecasting models. For ramp rate, Model 4 uses the selected $X \mid R$ condition as the best cp-WPRF model with the optimal Gumbel copula. For ramp magnitude, Model 4 uses the selected $X \mid R M S$ condition as the best cp-WPRF model with the optimal $t$ copula. For ramp duration, Model 4 uses the selected $X \mid D R$ condition as the best cp-WPRF model with the optimal Gaussian copula. For ramp start time, Model 4 uses the selected $X \mid D R S P$ condition as the best cp-WPRF model with the optimal $t$ copula. For simplicity of comparison, Figure 7 shows the coverage probabilities (reliability) and interval scores (sharpness) for different ramp features. As shown, the coverage probability curve of Model 4 (the blue solid line) is the closest to the ideal nominal proportion line (the red solid line) in all cases. It also shows that Model 4 has the smallest interval scores for each nominal proportion. Particularly, Model 4 performs much better in terms of the interval score for ramp rate, as shown in Figure 7d. This is probably because the stochastic dependence between the ramp rate feature and its uncertainty is simultaneously considered in Model 4. For a better illustration, Table 2 lists the numerical results of the evaluation metrics for different ramp features. Specifically, the reliability metric ranges of models $1,2,3$, and 4 are $4 \%-13 \%, 6 \%-$ $14 \%, 7 \%-13 \%$, and $1 \%-7 \%$, respectively. The sharpness metric ranges of models $1,2,3$, and 4 are $40 \%-134 \%, 35 \%-63 \%, 31 \%-69 \%$, and 30\%-51\%, respectively. The final SEM ranges are $0.3-1.04,0.09-0.28,-0.15-0.42$, and $-1.14--0.85$, respectively. For all four ramp features, Model 4 presents the smallest ACE, ASV, and SEM values. This is because Model 4 considers both the wind power ramp forecasting error uncertainty and the stochastic dependence of different ramp features, unlike both Model 1 and Model 2. In addition, the accurate characterization of marginal 
distributions in Model 4 can significantly improve probabilistic wind power ramp forecasting metrics compared to Model 3.

Table 2. Comparative Results for Different Ramp Features

\begin{tabular}{llrrrr}
\hline Features & Metrics & Model 1 & Model 2 & Model 3 & Model 4 \\
\hline \multirow{4}{*}{ Rate } & ACE [\%] & 12.46 & 6.64 & 9.45 & 1.64 \\
& ASV [\%] & 40.18 & 38.97 & 31.58 & 30.05 \\
& SEM & 1.02 & 0.27 & -0.15 & -1.14 \\
\hline \multirow{4}{*}{ Mag. } & ACE [\%] & 4.87 & 8.40 & 7.53 & 2.83 \\
& ASV [\%] & 134.30 & 63.03 & 68.74 & 50.26 \\
& SEM & 0.53 & 0.28 & 0.18 & -0.99 \\
\hline \multirow{4}{*}{ Dur. } & ACE [\%] & 6.86 & 13.25 & 12.86 & 6.34 \\
& ASV [\%] & 65.76 & 35.98 & 46.13 & 34.48 \\
& SEM & 0.30 & 0.12 & 0.42 & -0.85 \\
\hline \multirow{3}{*}{ Start } & ACE [\%] & 9.37 & 9.35 & 8.85 & 5.89 \\
Time & ASV [\%] & 110.80 & 45.35 & 46.24 & 34.82 \\
& SEM & 1.04 & 0.09 & -0.04 & -1.09 \\
\hline
\end{tabular}



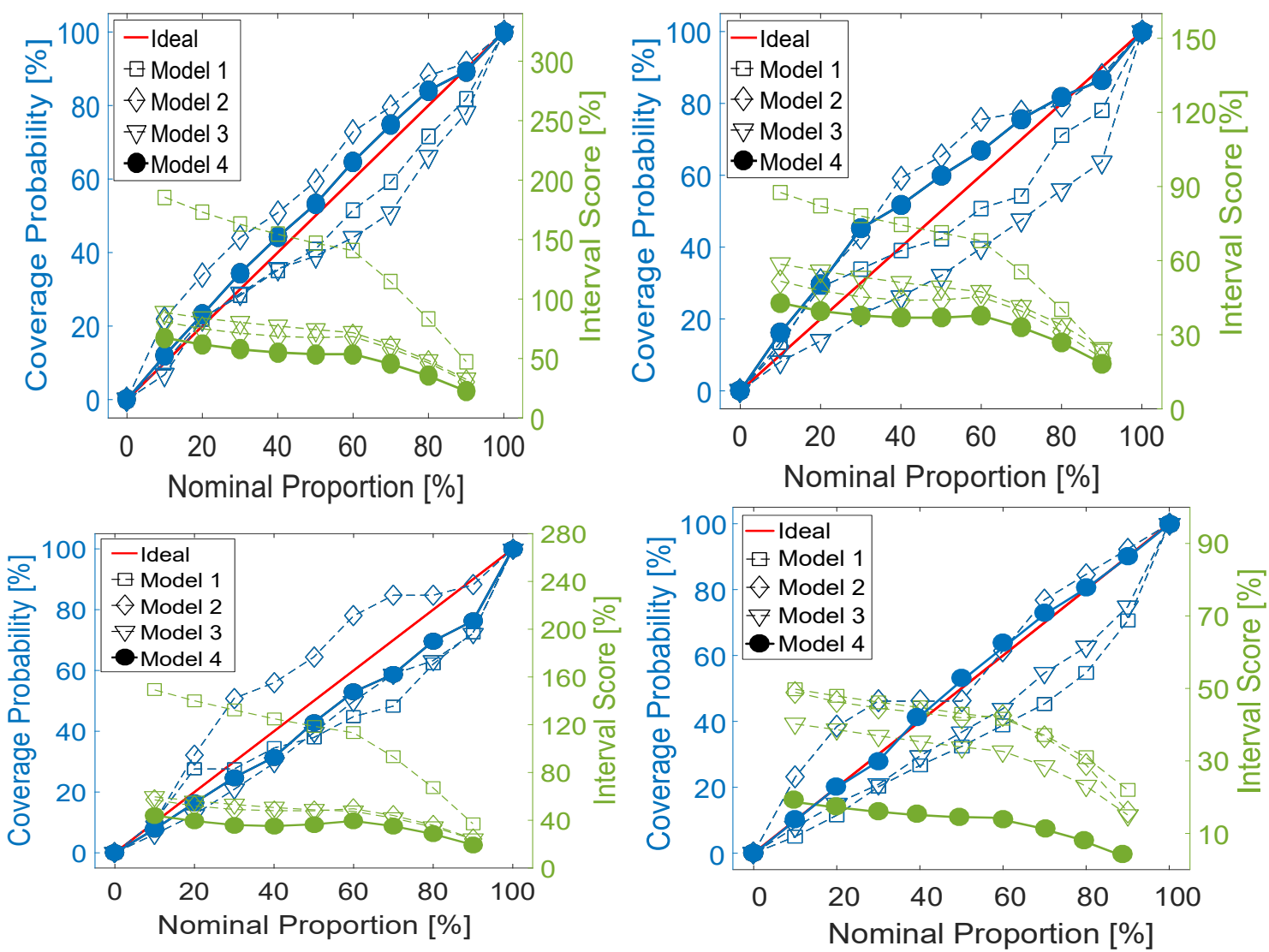

Figure 7. Comparison of different models of conditional probabilistic forecasts for ramp features in the Dallas area. From left to right: (a) magnitude, (b) duration, (c) start time, and (d) rate

The impact of the copula-based stochastic dependence is further analyzed by comparing Model 2 and Model 4. For all wind power ramp features, evaluation metrics are better with improved SEM values of $1.41[=0.27-(-1.14)], 1.27[=0.28-(-0.99)], 0.97[=0.12-(-0.85)]$, and $1.18[=0.09$ $(-1.09)]$. These findings would help balancing authorities efficiently manage wind power ramps. For example, a better forecast accuracy of ramp magnitude can be used to design more reliable ramping products in the electricity market.

Case 2: The ramp rate data of the four regions in Miami, Chicago, New York, and Los Angeles are used to verify the robustness of the developed cp-WPRF model. Figure 8 shows the coverage probabilities and interval scores for ramp rate forecasts in these regions. Model 4 presents the closest coverage probability curve (the blue solid line) to the ideal nominal proportion line (the red solid line) in all cases. This model also shows the smallest interval scores for each nominal proportion. Numerical results of evaluation metrics for ramp rate in different regions are illustrated in Table 3. Specifically, the reliability metric ranges of models $1,2,3$, and 4 are $2 \%-$ $14 \%, 3 \%-7 \%, 4 \%-8 \%$, and $2 \%-3 \%$, respectively. The sharpness metric ranges of models $1,2,3$, and 4 are $33 \%-37 \%, 31 \%-47 \%, 26 \%-33 \%$, and $25 \%-32 \%$, respectively. The SEM ranges are 0.37-1.16, -0.06-0.5, -0.65-0.48, and -0.91--0.79, respectively. For all regions, Model 4 presents the smallest ACE, ASV, and SEM values. This is because Model 4 considers both the wind power ramp forecasting error uncertainty and the stochastic dependence of other ramp features. Comparing Model 2 to Model 4 shows significant improvements to all four regions in the evaluation metrics. The improved SEM values in Miami, Chicago, New York, and Los 
Angeles are $1.31[=0.40-(-0.91)], 1.39[=0.50-(-0.89)], 1.27[=0.41-(-0.86)]$, and $0.73[=-0.06-(-$ $0.79)$ ], respectively.
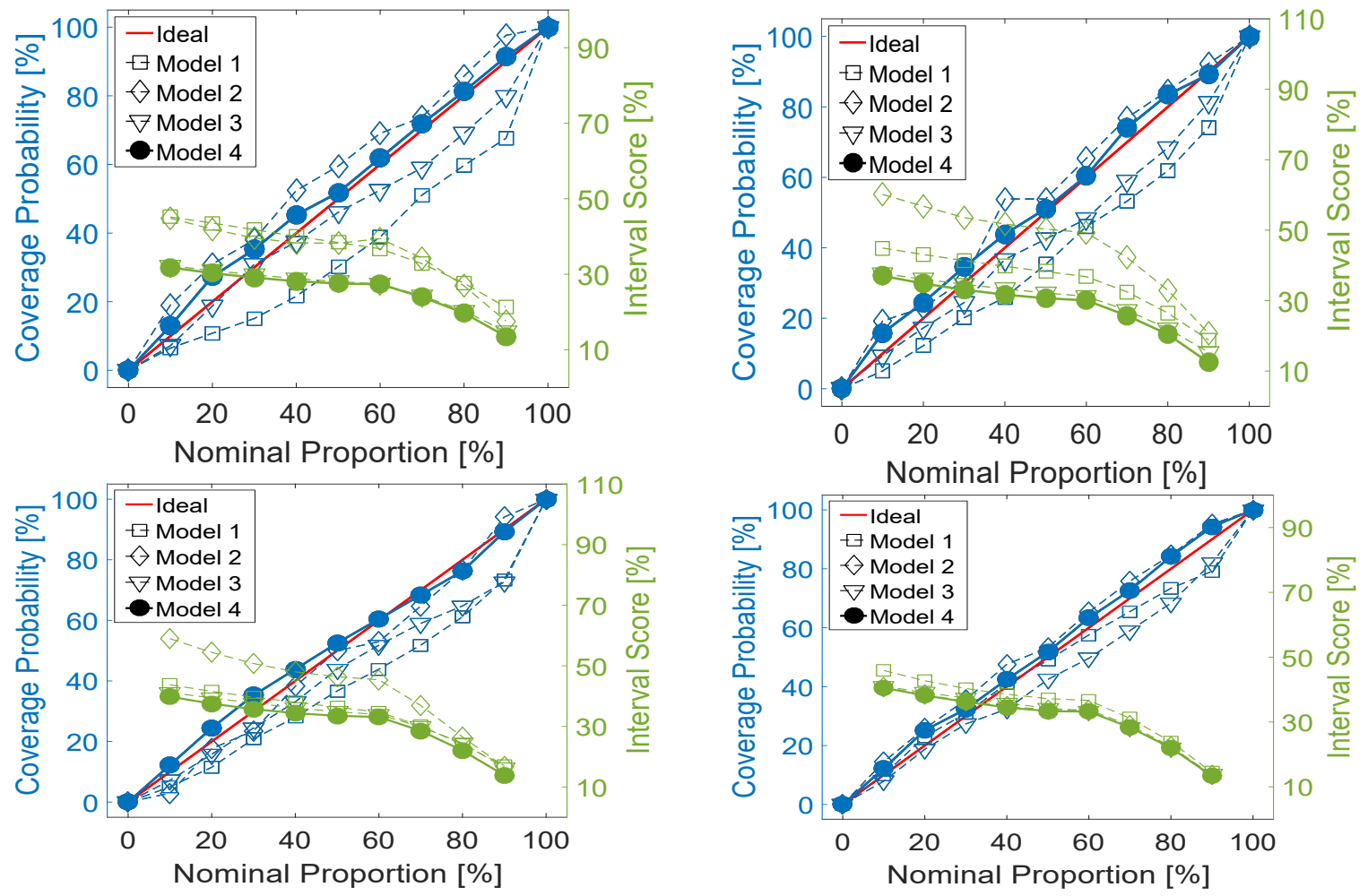

Figure 8. Comparison of different models of $c p-W P R F$ for ramp rate in multiple regions. From left to right: (a) Miami, (b) Chicago, (c) New York City, and (d) Los Angeles 
Table 3. Comparative Results for Ramp Rate in Multiple Regions

\begin{tabular}{llllll}
\hline Regions & Metrics & Model 1 & Model 2 & Model 3 & Model 4 \\
\hline \multirow{3}{*}{ MIA } & ACE [\%] & 13.51 & 6.93 & 4.65 & 2.65 \\
& ASV [\%] & 36.38 & 35.67 & 26.31 & 25.71 \\
& SEM & 1.16 & 0.40 & -0.65 & -0.91 \\
\hline \multirow{3}{*}{ CHI } & ACE [\%] & 10.56 & 4.55 & 5.72 & 2.61 \\
& ASV [\%] & 35.78 & 46.42 & 29.97 & 28.45 \\
& SEM & 0.73 & 0.50 & -0.34 & -0.89 \\
\hline \multirow{3}{*}{ NYC } & ACE [\%] & 10.69 & 3.42 & 7.03 & 2.27 \\
& ASV [\%] & 33.91 & 42.67 & 32.65 & 30.93 \\
& SEM & 0.53 & 0.41 & -0.07 & -0.86 \\
\hline \multirow{2}{*}{ LA } & ACE [\%] & 2.78 & 4.33 & 5.66 & 2.58 \\
& ASV [\%] & 34.55 & 31.45 & 31.71 & 31.07 \\
& SEM & 0.37 & -0.06 & 0.48 & -0.79 \\
\hline
\end{tabular}

Another interesting finding is that the interval score difference between Model 3 and Model 4 in Figure 7 is more significant than that in all four regions in Figure 8. This is because the GMM marginal distribution fits the wind power ramp forecasting error uncertainties significantly better than the normal marginal distribution. For the other four regions in Figure 8, however, the GMM marginal distribution fits the wind power ramp forecasting error uncertainties only slightly better than the normal marginal distribution. To compare the fitting performance of the normal and GMM distributions for wind power ramp forecasting errors, Table 4 illustrates the Chisquare $\left(\chi^{2}\right)$ statistics to measure the goodness of fit. As shown, for the Dallas data, the fitting performance of GMM is about $72 \%$ better than that of the normal distribution. For the other four regions (MIA, CHI, NYC, and LA), however, the slight improvements of using GMM are approximately $21 \%-27 \%$, compared to the normal distribution.

Table 4. $\chi^{2}$ Statistics Comparison of GMM and Normal Distributions for Ramp Rate at Five Regions

\begin{tabular}{lccccc}
\hline Distributions & MIA & CHI & NYC & LA & DAL \\
\hline Normal & 3.11 & 2.98 & 3.28 & 3.31 & 3.82 \\
GMM & 2.37 & 2.15 & 2.62 & 2.54 & 1.07 \\
Improvement by GMM & $23 \%$ & $27 \%$ & $21 \%$ & $23 \%$ & $72 \%$ \\
\hline
\end{tabular}

\subsubsection{Robustness Analysis of cp-WPRF Models}

To verify the robustness of the developed model with different forecasting methods, three autoregressive moving average model (ARMA) methods are used as a comparison with the support vector machine-based cp-WPRF model in Figure 7d: $\operatorname{ARMA}(1,1), \operatorname{ARMA}(2,1)$, and ARMA(3,1). Figure 9 shows the performance of the cp-WPRF models using various ARMA forecasting methods. As shown in Figure 9a-c, the developed cp-WPRF model (Model 4) 
consistently presents the closest coverage probability curve (the blue solid line) and the smallest interval score (the green solid line) when using different ARMA methods. Specifically, Figure $9 \mathrm{~d}$ compares the SEM values of the cp-WPRF models using different forecasting methods. The developed cp-WPRF model (Model 4) consistently provides the smallest SEM values (see the dark blue bars). These observations verify that the developed cp-WPRF is robust with different forecasting methods.

Table 5 illustrates the optimal copula models and best conditions of different forecasting methods based on the cp-WPRF models. It shows that the optimal copula model might change with the forecasting method. This is because the probability distributions of the ramp rates forecasted by the distinct forecasting method are different. These probability distributions could generate different cumulative distribution function values of $u=F\left(x_{r}\right)$, which can impact the estimated parameters and the performance of the optimal copula model with different Bayesian information criterion values in Eq. (13).
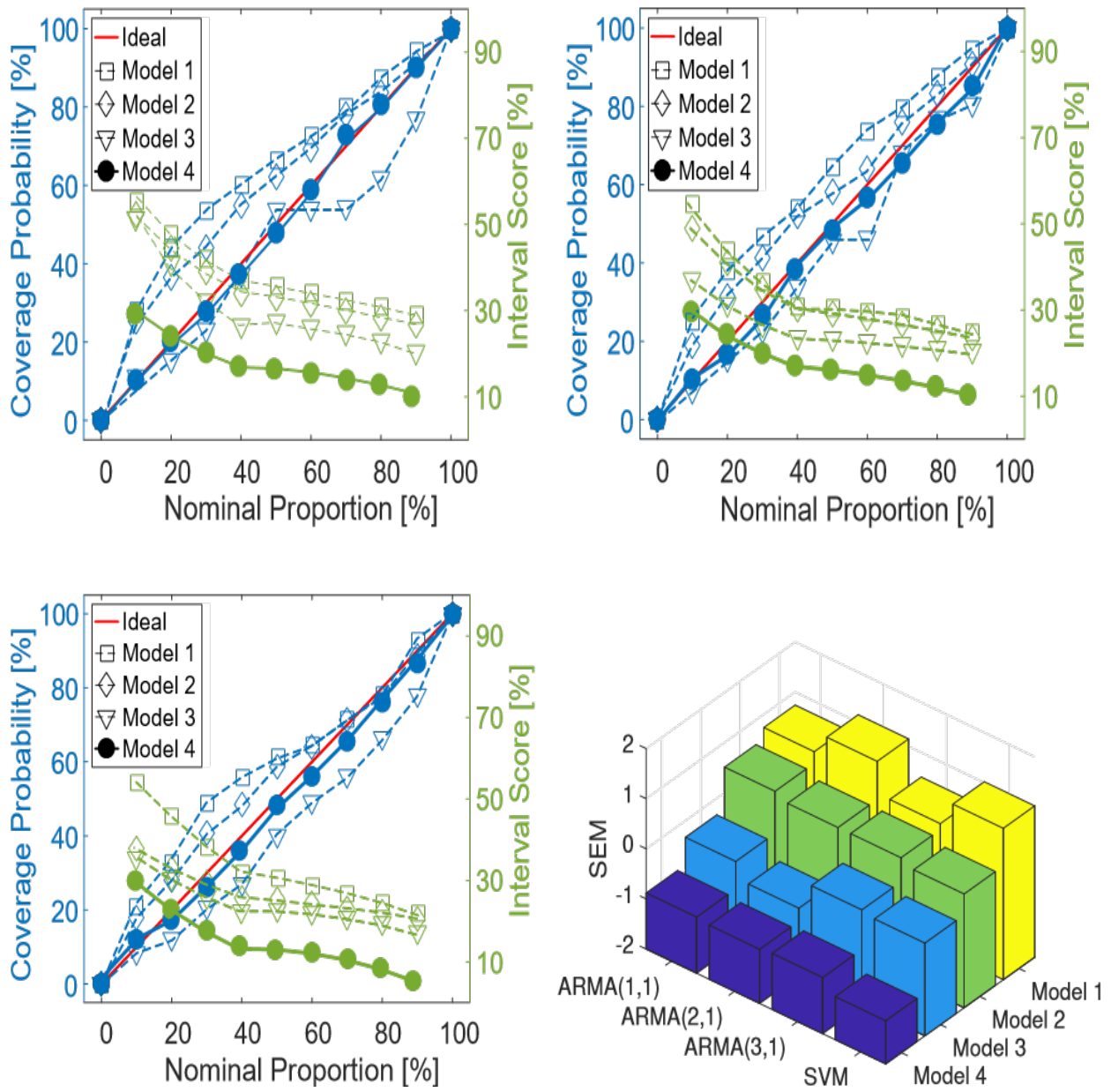

Figure 9. Performance of different forecasting methods based cp-WPRF models (from left to right): ARMA-based cp-WPRF in (a), (b), (c); SEM values of multiple models in (d) 
Table 5. Optimal Copula Models and Best Conditions of Different Forecasting Methods-Based cp-WPRF Models

\begin{tabular}{ccc}
\hline Forecasting Method & Best Condition & Optimal Copula Model \\
\hline $\operatorname{ARMA}(1,1)$ & $X \mid R D M$ & $\mathrm{t}$ \\
$\operatorname{ARMA}(2,1)$ & $X \mid R M S$ & Frank \\
$\operatorname{ARMA}(3,1)$ & $X \mid M R$ & $\mathrm{t}$ \\
$\begin{array}{c}\text { Support Vector } \\
\text { Machine }\end{array}$ & $X \mid R$ & Gumbel \\
\hline
\end{tabular}




\section{Sequential Multi-Timescale Electricity Market Simulation Tool (OpenSMEMS)}

The objective of the sequential market simulation tool is to represent market events in their practical order of occurrence to analyze the intrinsic implications of one event on another. The analysis of the impact of wind ramping product is a special point of interest. To this end, we model the day-ahead market problem as a security-constrained unit commitment (SCUC) problem and the real-time market as a sequence of hour-ahead SCUC and intra-hour securityconstrained economic dispatch (SCED). Modeling details are presented in the following subsections.

\subsection{Security-Constrained Unit Commitment}

On a daily basis, electric power system operators face the challenge of ensuring resource adequacy. This includes securing enough generation capacity to supply the next day's estimated or forecasted demand. Day-ahead SCUC is used to achieve this goal and schedule for each hour of the next day the generation units that should come online for reliable and economic operation of the grid. The objective function and constraints that define the SCUC model are presented in Eq. (2.1) through Eq. (2.27). The SCUC problem seeks to minimize the overall operating cost $T C$, comprising startup costs $\left(s u_{g, t}\right)$ and shutdown costs $\left(s d_{g, t}\right)$, production costs $\left(p c_{g, t}\right)$, ramping costs $\left(r c_{g, t}\right)$ and load-shedding costs $\left(l s_{t}\right)$, as expressed by Eq. (2.1). Equations (2.2)(2.6) define the aforementioned components of $T C$.

$$
T C=\sum_{t \in T}\left(\sum_{g \in G} s u_{g, t}+s d_{g, t}+p c_{g, t}+r c_{g, t}\right)+l s_{t}
$$

with:

$$
\begin{gathered}
s u_{g, t} \geq S U_{g}\left(v_{g, t}-v_{g, t-1}\right), \forall g \in G_{T h}, t \in T \\
s d_{g, t} \geq S D_{g}\left(v_{g, t-1}-v_{g, t}\right), \forall g \in G_{T h}, t \in T \\
p c_{g, t}=N L_{g} v_{g, t}+\sum_{k=1}^{K_{g}} p_{g, t}^{k} C_{g}^{k}, \forall g \in G_{T h}, t \in T \\
r c_{g, t}=R U_{g} f r u_{g, t, s}+R D_{g, t} f r d_{g, t, s}, \forall g \in G, t \in T, s \in S \\
l s_{t}=\sum_{b \in B_{L}} L S P_{b} \delta_{b, t}, \forall t \in T
\end{gathered}
$$

Equations (2.2) and (2.3) define the units' startup and shutdown costs, respectively; Eq. (2.4) presents the generation production cost modeled as a combination of no-load costs and blockwise generator cost functions $C_{g}^{k}$, where $k$ is the block index. $C_{g}^{k}$ is the amount it costs generator $\mathrm{g}$ to produce one unit of energy when operating in block $k$. We assume $C_{g}^{k}$ increases with $k$, i.e., $0 \leq C_{g}^{k}<C_{g}^{k+1}$. Equation (2.5) gives the system ramping product procurement cost. We use Eq. 
(2.6) to express the load curtailment penalty, where we consider a locational curtailment penalty $L S P_{b}$, specific to each load bus $b$. In addition to the system operating cost definition, several operational constraints need to be enforced to ensure feasible, reliable, and secure grid operations. These well-known and widely used constraints are presented in Eq. (2.7) through Eq. (2.27), for completeness.

In power generation industry practice, the operation cycles of the generation units are tracked. Different classes of generation assets have different up- and down-time requirements. Constraints (2.7) through (2.12) ensure that these requirements are met.

$$
\begin{gathered}
\sum_{t=1}^{I U T_{g}}\left(1-v_{g, t}\right)=0, \forall g \in G_{T h} \\
\sum_{\tau=t}^{t+U T_{g}-1} v_{g, \tau} \geq U T_{g}\left(v_{g, t}-v_{g, t-1}\right), \forall g \in G_{T h}, t=I U T_{g}+1, \ldots,|T|-U T_{g}+1 \\
\sum_{\tau=t}^{T}\left[v_{g, \tau}-\left(v_{g, t}-v_{g, t-1}\right)\right] \geq 0, \forall g \in G_{T h}, t=|T|-U T_{g}+2, \ldots,|T| \\
\sum_{t=1}^{I D T_{g}} v_{g, t}=0, \forall g \in G_{T h} \\
\sum_{\tau=t}^{T}\left(1-v_{g, \tau}\right) \geq D T_{g}\left(v_{g, t-1}-v_{g, t}\right), \forall g \in G_{T h}, t=I D T_{g}+1, \ldots,|T|-D T_{g}+1 \\
\sum_{\tau=t}^{T}\left[1-v_{g, \tau}-\left(v_{g, t-1}-v_{g, t}\right)\right] \geq 0, \forall g \in G_{T h}, t=|T|-D T_{g}+2, \ldots,|T|
\end{gathered}
$$

To ensure ramping, regulating, and spinning reserves can be procured, constraints (2.13) define their joint feasible region. Constraints (2.14) limit the available power in the time interval prior to a shutdown, to the shutdown rate $R_{g}^{S D}$. Equation (2.15) controls the amount of spinning reserve offered by generation unit $g$ at time $t$, and Eq. (2.16) enforces its ramp capability limits.

$$
\begin{gathered}
\bar{p}_{g, t}-p_{g, t-1}-r r_{g, t}^{U}-s r_{g, t} \leq R_{g}^{U} v_{g, t-1}+R_{g}^{S U}\left(v_{g, t}-v_{g, t-1}\right) \\
+P_{g}^{\max }\left(1-v_{g, t}\right), \forall g \in G_{T h}, t \in T \\
\bar{p}_{g, t} \leq R_{g}^{S D} v_{g, t}+P_{g}^{\max } v_{g, t+1}, \forall g \in G_{T h}, t=1, \ldots,|T|-1 \\
p_{g, t}+s r_{g, t} \leq \bar{p}_{g, t}, \forall g \in G, t \in T
\end{gathered}
$$




$$
p_{g, t-1}-p_{g, t} \leq R_{g}^{D} v_{g, t}+R_{g}^{S D}\left(v_{g, t-1}-v_{g, t}\right)+P_{g}^{\max }\left(1-v_{g, t-1}\right), \forall g \in G_{T h}, t \in T
$$

The amount of spinning and regulating reserve procured at a given time depends on the capabilities of individual generation units and the overall reserve requirements. These requirements are generally determined based on estimated demand profiles. Equations (2.17) through (2.22) express such constraints. Equations (2.17), (2.18), and (2.19) ensure that the estimated requirements for spinning, regulation-up, and regulation-down reserves are met, respectively. Constraints (2.20) through (2.22) ensure that each participating generation unit is capable of providing the amount of reserve it offers.

$$
\begin{aligned}
& \sum_{g \in G} s r_{g, t} \geq S R R_{t}, \forall t \in T \\
& \sum_{g \in G} r r_{g, t}^{U} \geq R R R_{t}^{U}, \forall t \in T \\
& \sum_{g \in G} r r_{g, t}^{D} \geq R R R_{t}^{D}, \forall t \in T \\
& s r_{g, t} \leq R_{g}^{U}, \forall g \in G, t \in T \\
& r r_{g, t}^{U} \leq R_{g}^{U}, \forall g \in G, t \in T \\
& r r_{g, t}^{D} \leq R_{g}^{D}, \forall g \in G, t \in T
\end{aligned}
$$

The power output of unit $g$ is defined, in Eq. (2.23), as the combination of its minimum production $P_{g}^{\text {min }}$ and its generation amount in each block $k$. In fact, we slice the generation margin into blocks with size $\Delta P_{g}^{k}$, as shown in constraint (2.24).

$$
\begin{gathered}
p_{g, t}=P_{g}^{m i n} v_{g, t}+\sum_{k=1}^{K_{g}} p_{g, t}^{k}, \forall g \in G_{T h}, t \in T \\
0 \leq p_{g, t}^{k} \leq \Delta P_{g}^{k}, \forall g \in G, t \in T
\end{gathered}
$$

Constraints (2.25) and (2.26) impose the minimum and maximum generation dispatch limits.

$$
\begin{gathered}
P_{g}^{\min } v_{g, t} \leq p_{g, t} \leq \bar{p}_{g, t}, \forall g \in G_{T h}, t \in T \\
0 \leq \bar{p}_{g, t} \leq P_{g}^{\max } v_{g, t}, \forall g \in G_{T h}, t \in T
\end{gathered}
$$

Constraints (2.27) and (2.28) define the maximum available flexible ramping-up and rampingdown products, respectively. 


$$
\begin{gathered}
p_{g, t}+f r u_{g, t, s} \leq \bar{p}_{g, t+1}, \forall g \in G, t=1, \ldots,|T|-1, s \in S \\
p_{g, t}-f r d_{g, t, s} \geq P_{g}^{m i n} v_{g, t+1}, \forall g \in G_{T h}, t=1, \ldots,|T|-1, s \in S
\end{gathered}
$$

For renewable generation units, the average hourly available power output is constrained by the forecasted power as expressed in Eq. (2.29). Constraint (2.30) bounds $g$ 's actual output dispatch.

$$
\begin{gathered}
0 \leq \bar{p}_{j, t} \leq \frac{1}{|S|} \sum_{s \in S} F O R_{g, t, s}, \forall g \in G_{R e}, t \in T \\
0 \leq p_{g, t} \leq \bar{p}_{g, t}, \forall g \in G_{R e}, t \in T
\end{gathered}
$$

In addition to these constraints, system constraints that account for supply-demand balance (2.31) and transmission line limits (2.32) are worth enforcing.

$$
\begin{gathered}
\sum_{g \in G} p_{g, t}-\sum_{b \in B_{L}} D_{b, t}-\delta_{b, t}=0, \forall t \in T \\
- \text { Limit }_{l} \leq \sum_{i \in B_{G}} G S F_{l-i} p_{i, t}^{i n j}-\sum_{b \in B_{L}} G S F_{l-b}\left(D_{b, t}-\delta_{b, t}\right) \leq \text { Limit }_{l}, \forall t \in T, l \in L
\end{gathered}
$$

where $p_{i, t}^{i n j}$ is the total power injected (by generation units connected) at generator bus $i$ in time $t, D_{b, t}$ the total demand (by all loads connected) at load bus $b$ in time $t$. Constraints (2.32) ensure that line flows do not exceed transmission capacities.

The amount of load curtailment at bus $b\left(\delta_{b, t}\right)$ is bound by the actual demand $D_{b, t}$ as in (2.33).

$$
\delta_{b, t} \leq D_{b, t}, \forall b \in B_{L}, t \in T
$$

Special attention is given to the wind ramping product formulation in [Sedzro 2018]. We opt for the wind ramping product formulation given by (2.34) and (2.35). These constraints are the equivalents of (2.27) and (2.28) for the wind ramping product. The up-ramping product captures both intra-time slot and inter-time slot ramps.

$$
\begin{gathered}
p_{w, t}+f r u_{w, t, s} \leq \max \left\{\bar{p}_{w, t}, \bar{p}_{w, t+1}\right\}, \forall w \in G_{W}, t \in T, s \in S \\
p_{w, t}-f r d_{w, t, s} \geq 0, \forall w \in G_{W}, t \in T, s \in S
\end{gathered}
$$

Equations (2.36) and (2.37) enforce ramp sufficiency. This set of constraints ensures that enough ramp reserve is available to meet the expected demand variability.

$$
\sum_{g \in G_{T h} \cup G_{W}} f r u_{i, t, s} \geq F R R_{t, s}^{U}, \forall t \in T, s \in S
$$




$$
\sum_{g \in G_{T h} \cup G_{W}} f r d_{i, t, s} \geq F R R_{t, s}^{D}, \forall t \in T, s \in S
$$

\subsection{Security-Constrained Economic Dispatch}

We formulate the SCED model as the linear counterpart of the SCUC by setting generation unit availability parameters $V_{g, t}$ to the same values as the corresponding SCUC unit status binary variables $v_{g, t}$. In other words, the unit status binary variable $v_{g, t}$ is replaced by the availability parameter $V_{g, t}$ in equations (2.2), (2.3), (2.13), (2.14), (2.16), (2.23), (2.25), ( 2.26), (2.28), in the SCED model. The SCED model is deployed both in the day-ahead market and in the realtime market.

In the day-ahead, the goal of the day-ahead SCED (DA-SCED) instance is to obtain the locational marginal prices. Hence, the linearization of the SCUC model using commitment status solutions from DA-SCUC, as discussed, is enough. The time horizon is the same as in SCUC, 24 $\mathrm{h}$ at an hourly time interval with 10-min renewable forecast resolution.

In real time, the aim of the real-time SCED (RT-SCED) is to evaluate the delivery of energy commitments in real time. Hence, ramping products are not considered in the RT-SCED. In addition, because RT-SCED is solved one time slot at a time, all RT-SCED variables and parameters are time-slot based. For example, the generation dispatch variable $p_{g, t}$ in SCUC becomes $p_{g, t, s}$ in RT-SCED.

\subsection{Market Simulation Sequence}

The sequential market simulation starts with the day-ahead unit commitment and ends with the real-time economic dispatch of the last time slot of the day, as shown in Figure 10. DA-SCUC and DA-SCED are solved for Tda hours. RT-SCUC is solved for Trt consecutive hours, i.e., $(\operatorname{Tr} t-1)$ look-ahead horizon, and produces hourly generation dispatch schedules and $\frac{60}{|S|}$-min ramp schedules. The order of a real-time market stage or run stg in the simulation sequence is given by (2.38). From (2.38), we derive the total number of runs given by (2.39). The stage time slot $s_{\text {stg }}$ is set to 0 for SCUC stages.

$$
\begin{aligned}
& O(s t g)=\left(\begin{array}{l}
2+t_{s t g}+\left(t_{s t g}-1\right)|S|+s_{s t g}, \forall t_{s t g} \leq T d a-\operatorname{Tr} t+1, s_{s t g} \in S \\
3+T d a-\operatorname{Tr} t+\left(t_{s t g}-1\right)|S|+s_{s t g}, \forall t_{s t g} \geq \operatorname{Tda}-\operatorname{Tr} t+1, s_{s t g} \in S
\end{array}\right. \\
& \text { Number }- \text { of }- \text { runs }=3+\operatorname{Tda}(|S|+1)-\operatorname{Tr} t
\end{aligned}
$$

Subsections 2.3.1 through 2.3.4 present the summary of the model components and specifics of the market stages shown in Figure 10. 


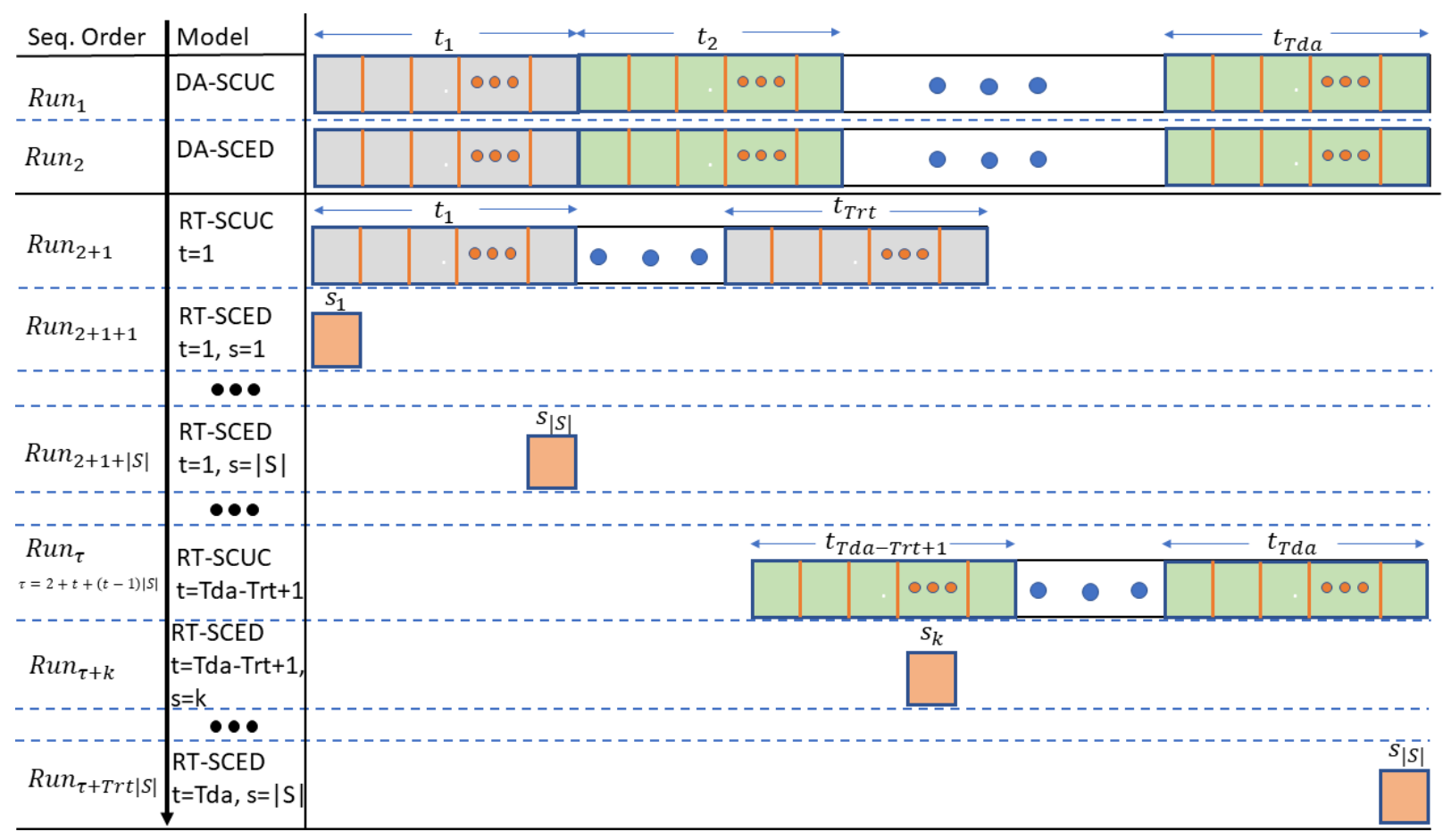

Figure 10. Market sequence

\subsubsection{DA-SCUC}

The objective of the DA-SCUC is to minimize the total system cost defined in Eq. (2.1), i.e.:

$$
\min T C
$$

subject to the following set of constraints: (2.2) through (2.28).

\subsubsection{DA-SCED}

As mentioned in Section 2.1.2, the purpose of the DA-SCED stage is to compute the day-ahead locational marginal price values. Hence, the objective of our DA-SCED model is the same as in Eq. (2.40).

The set of constraints enforced are (2.2) through (2.28), with the following modification applied to constraints (2.2), (2.3), (2.13), (2.14), (2.16), (2.23), (2.25), (2.26), and (2.28): the unit status binary variable $v_{g, t}$ is replaced by the availability parameter $V_{g, t}$, which is a solution from the DA-SCUC stage.

\subsubsection{RT-SCUC}

As shown in Figure 1, the RT-SCUC stage solves the unit commitment problem of minimizing the total system cost as in (2.40), subject to constraints (2.2) through (2.28). The main difference is the reduction of the time horizon from $T d a$ to $T r t$, where $T d a$ is the length of the set of time interval $T$ considered in the DA-SCUC, and Trt is that of the time interval set considered in RTSCUC. 


\subsubsection{RT-SCED}

The RT-SCED stage solves for each time slot $s$ in a given time interval $t$, one time slot at a time. Therefore, an RT-SCED stage is defined by its time interval and time slot, as shown in Figure 10. For clarity, we present the complete RT-SCED model:

Subject to:

$$
\min \sum_{g \in G} p c_{g, t, s}+l s_{t, s}
$$

$$
\begin{aligned}
& p c_{g, t, s}=\frac{1}{|S|}\left(N L_{g} V_{g, t}+\sum_{k=1}^{K_{g}} p_{g, t, s}^{k} C_{g}^{k}\right), \forall g \in G_{T h}, t \in T, s \in S \\
& l s_{t}=\frac{1}{|S|}\left(\sum_{b \in B_{L}} L S P_{b} \delta_{b, t, s}\right), \forall t \in T \\
& p_{g, t, s}=P_{g}^{\min } V_{g, t}+\sum_{k=1}^{K_{g}} p_{g, t, s}^{k}, \forall g \in G_{T h}, t \in T, s \in S \\
& 0 \leq p_{g, t, s}^{k} \leq \Delta P_{g}^{k}, \forall g \in G_{T h}, t \in T, s \in S \\
& P_{g}^{\min } V_{g, t} \leq p_{g, t} \leq \bar{p}_{g, t, s}, \forall g \in G_{T h}, t \in T, s \in S \\
& 0 \leq \bar{p}_{g, t, s} \leq P_{g}^{\max } V_{g, t}, \forall g \in G_{T h}, t \in T, s \in S \\
& 0 \leq \bar{p}_{g, t, s} \leq F O R_{g, t, s}, \forall g \in G_{R e}, t \in T, s \in S \\
& 0 \leq p_{g, t, s} \leq \bar{p}_{g, t, s}, \forall g \in G_{R e}, t \in T, s \in S \\
& \sum_{g \in G} p_{g, t, s}-\sum_{b \in B_{L}} D_{b, t, s}-\delta_{b, t, s}=0, \forall t \in T, s \in S \\
& - \text { Limit }_{l} \leq \sum_{i \in B_{G}} G S F_{l-i} p_{i, t, s}^{i n j}-\sum_{b \in B_{L}} G S F_{l-b}\left(D_{b, t, s}-\delta_{b, t, s}\right) \leq \text { Limit }_{l}, \forall t \in T, s \in S, l \in L \\
& \delta_{b, t, s} \leq D_{b, t, s}, \forall b \in B_{L}, t \in T, s \in S
\end{aligned}
$$

Constraints (2.42), (2.43), (2.44), (2.45), (2.46), (2.47), (2.48), (2.49), (2.50), (2.51), and (2.52) are, respectively, the SCED counterparts of (2.4), (2.6), (2.23), (2.24), (2.25), (2.26), (2.29), (2.30), (2.31), (2.32), and (2.33). 
In addition, because our RT-SCED solves for a single time slot at a time, we keep track of the previous dispatch to bound the current dispatch using the maximum ramp rate, as shown in constraints (2.53), where $P_{g, t, s-1}$ is the dispatch obtained from the previous RT-SCED stage and used in the current stage as a parameter.

$$
-\frac{1}{|S|} R_{g}^{D} \leq p_{g, t, s}-P_{g, t, s-1} \leq \frac{1}{|S|} R_{g}^{U}, \forall g \in G_{T h}, t \in T, s \in S
$$

Using the underlying models described in sections 2.1 and 2.3 as well as the simulation structure presented in this section, we developed OpenSMEMS. OpenSMEMS is fully developed in Python/Pyomo and tested using the solver "Xpress." Because of its modular design, it can be used to simulate market segments independently or in a sequential fashion. Because OpenSMEMS explicitly models the wind ramping product in market formulations, we present its native use case in Section 3. Nevertheless, it is possible to turn off the wind ramping product constraints in any market segment by a simple wind ramping product input option in the parameters. 


\section{OpenSMEMS Use Case: Evaluating the Impact of Wind Providing Ramping Services}

The aim of this case study is to evaluate the impact of wind ramping product in either the dayahead or real-time market segments. To this end, we model and simulate scenarios with and without the wind ramping product. Three main scenarios are considered:

- Scenario 0 (Sc.0): base case scenario where wind is not allowed to provide ramping services in either the day-ahead or real-time market

- Scenario 1 (Sc.1): scenario where wind provides ramping services in the day-ahead market segment only

- Scenario 2 (Sc.2): scenario where wind provides ramping services in the real-time market segment only.

We also study the sensitivity of the wind ramping product with regard to wind penetration levels, such as $10 \%, 20 \%, 30 \%, 40 \%$ and $50 \%$. These penetrations are tested on the PJM 5-bus system. To ensure that OpenSMEMS is scalable, we simulate the Texas A\&M University (TAMU) 2,000-bus system at 25\% wind penetration and for scenarios Sc.0 through Sc.2. Section 4 presents the simulation results and analysis.

This study does not reflect the details of any specific existing market. We consider a generalized electricity market structure made of day-ahead and real-time segments. The DA-SCUC and DASCED are 24-hour horizon market instances $(T d a=24)$, with hourly generation schedules and 10-min ramp services. The DA-SCED is a mere linearization of the DA-SCUC with the purpose of computing the locational marginal prices. The time horizon of the RT-SCUC is set to 3 hours $(\operatorname{Trt}=3)$, i.e, 1-hour operation and 2-h look-ahead. Each instance of RT-SCED covers a 10-min time slot, and we have six time slots in 1 hour $(|S|=6)$. With these settings, the total number of stages in a daily market sequence is 168 (see Eq. (2.39)).

Sections 3.1 and 3.2 present the test systems and their corresponding results.

\subsection{Modified PJM 5-Bus System}

The 5-bus system used for the wind ramping impact study is presented in Figure 11. The base case system comprises 1,219 MW of supply capacity with $6 \%$ solar photovoltaic, $20 \%$ wind, and $74 \%$ natural gas. The total load is 1,250 MW. Throughout the study, the capacity of the photovoltaic and natural gas assets is kept unchanged, whereas the capacity of wind power plants can be linearly scaled to achieve a desired wind penetration according to the formulae (3.1) defining the scaling factor.

$$
\sigma=\frac{\gamma^{n e w}}{\gamma^{\text {init }}} \times \frac{1-\gamma^{\text {init }}}{1-\gamma^{\text {new }}}
$$

where $\gamma^{\text {init }}$ is the initial wind penetration (e.g., 0.02 for $20 \%$ ), and $\gamma^{\text {new }}$ is the desired penetration. 


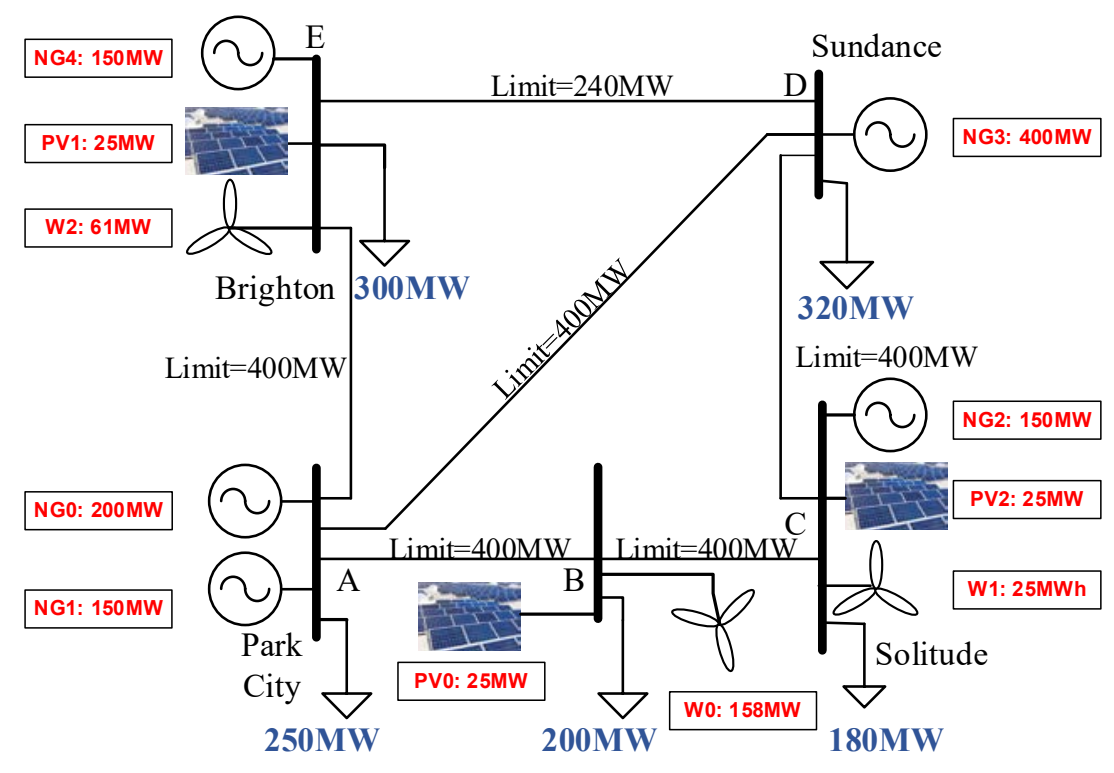

Figure 11. Modified PJM 5-bus test system

In addition to the network data required as input to the market simulation tool, load and wind power forecast data are necessary. Figure 12 shows load and wind power forecast profiles, as perceived in each market segment, for one day at $20 \%$ wind penetration.
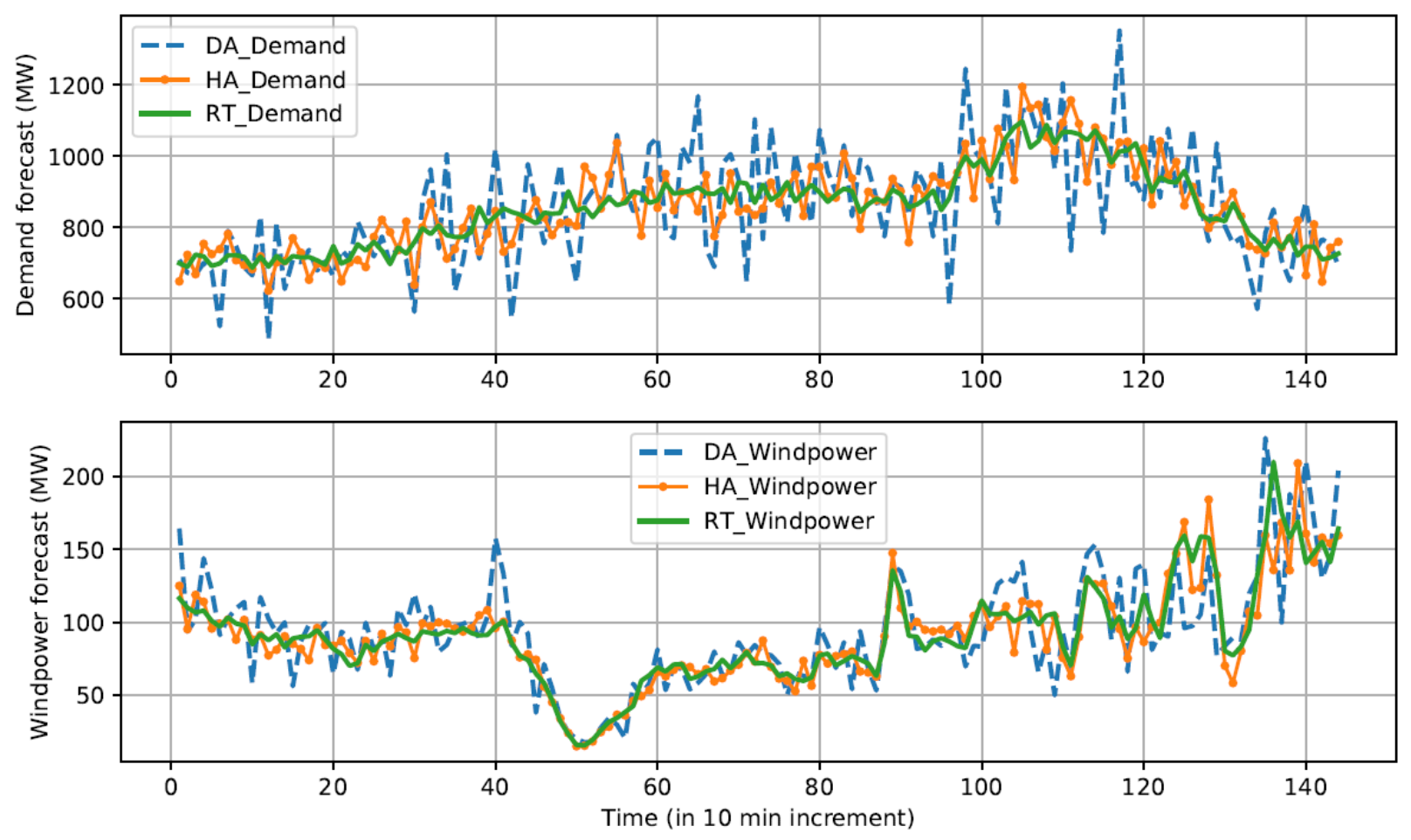

Figure 12. Demand and wind power forecasts at different market stages: PJM 5-bus

The "HA" or hour-ahead flag refers to the RT-SCUC stage, whereas the "RT" flag designates the RT-SCED stage. The "DA" flagged forecasts are used in both DA-SCUC and DA-SCED. 
At $20 \%$ wind power penetration in the legacy case, where the flexible ramping product is entirely provided by thermal units (i.e., natural gas units in this test system), OpenSMEMS gives the dispatch by fuel type shown in Figure 13.
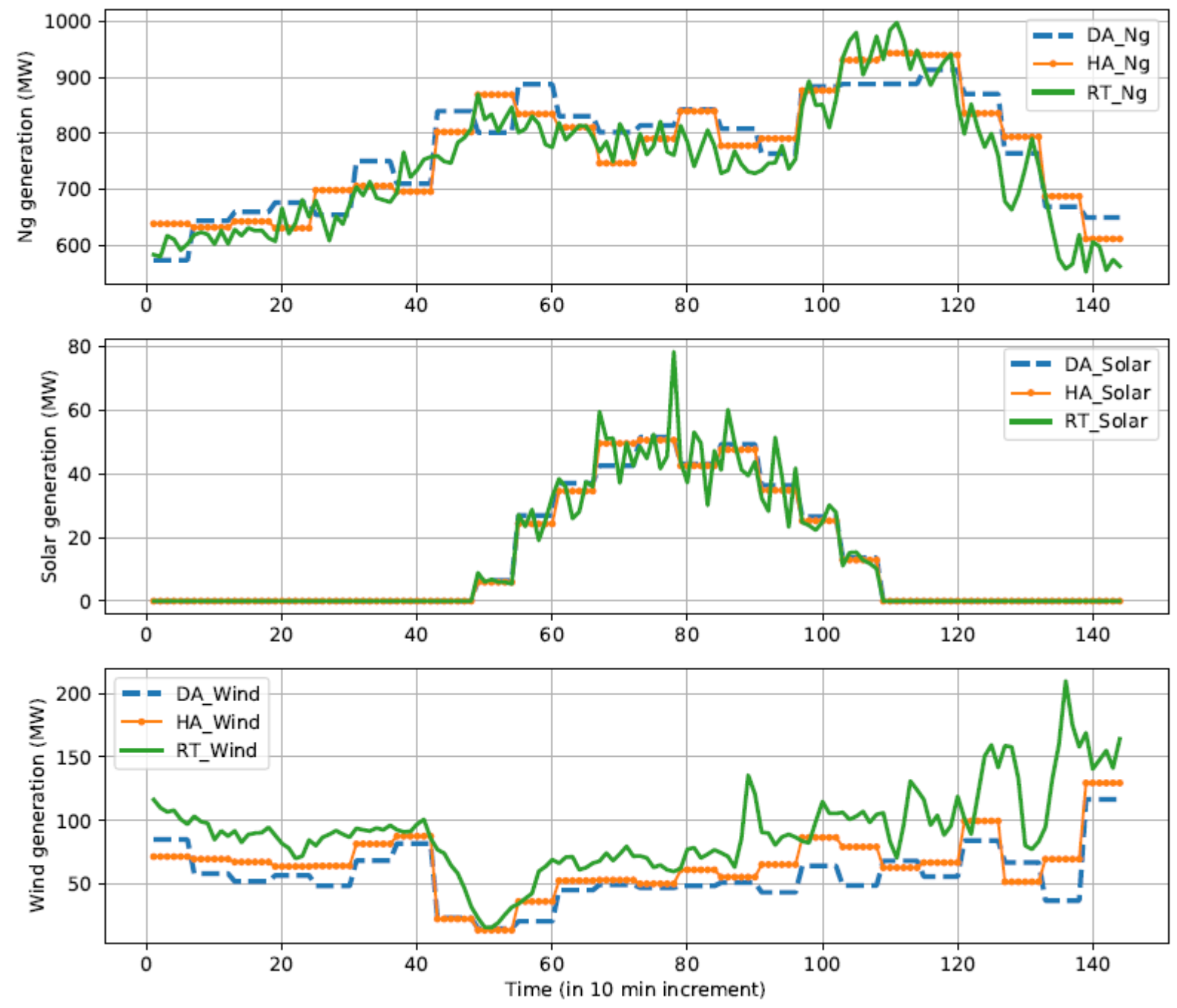

Figure 13. Dispatch by fuel type in different market segments in Sc.0

From one market stage to another (see Figure 10), demand and renewable output forecasts are refined, which leads to a better dispatch, as shown in Figure 13. Note that the DA-SCUC and RT-SCUC yield hourly schedules. With approximately the same wind power forecast trend and magnitude in all market segments, we see significantly different wind power generation profiles in the day-ahead and real-time commitments as well as in the real-time dispatch. This indicates intra- and inter-temporal wind curtailments that could be leveraged to provide ramping.

To evaluate the impact of the wind resources providing ramping product on the system ramp procurement cost, we compare the ramp cost in the base scenario (Sc.0, no wind ramping product) with the ramp cost in Scenario Sc.1 for the day-ahead and with Sc.2 for real time. We observe a ramping decrease in both the day-ahead and real time. To capture the trend of this impact, as we witness increasing wind penetration levels, we perform this analysis for wind 
levels ranging from $10 \%$ to $50 \%$. Figure 14 shows the temporal day-ahead ramp cost reductions for each wind penetration level, whereas Figure 15 presents the temporal real-time ramp cost reductions for each wind penetration.

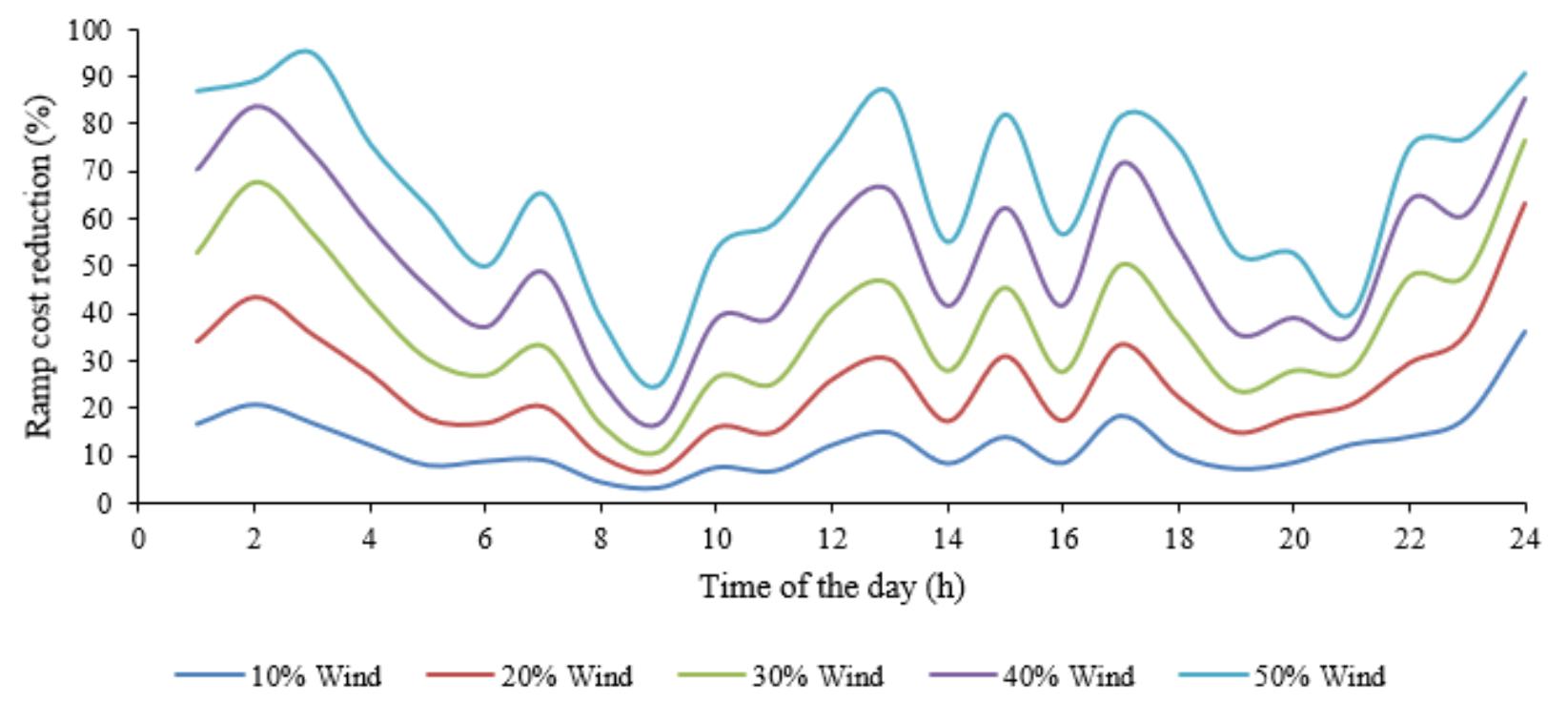

Figure 14. Impact of wind ramping product on system day-ahead ramp procurement cost at different wind penetration levels

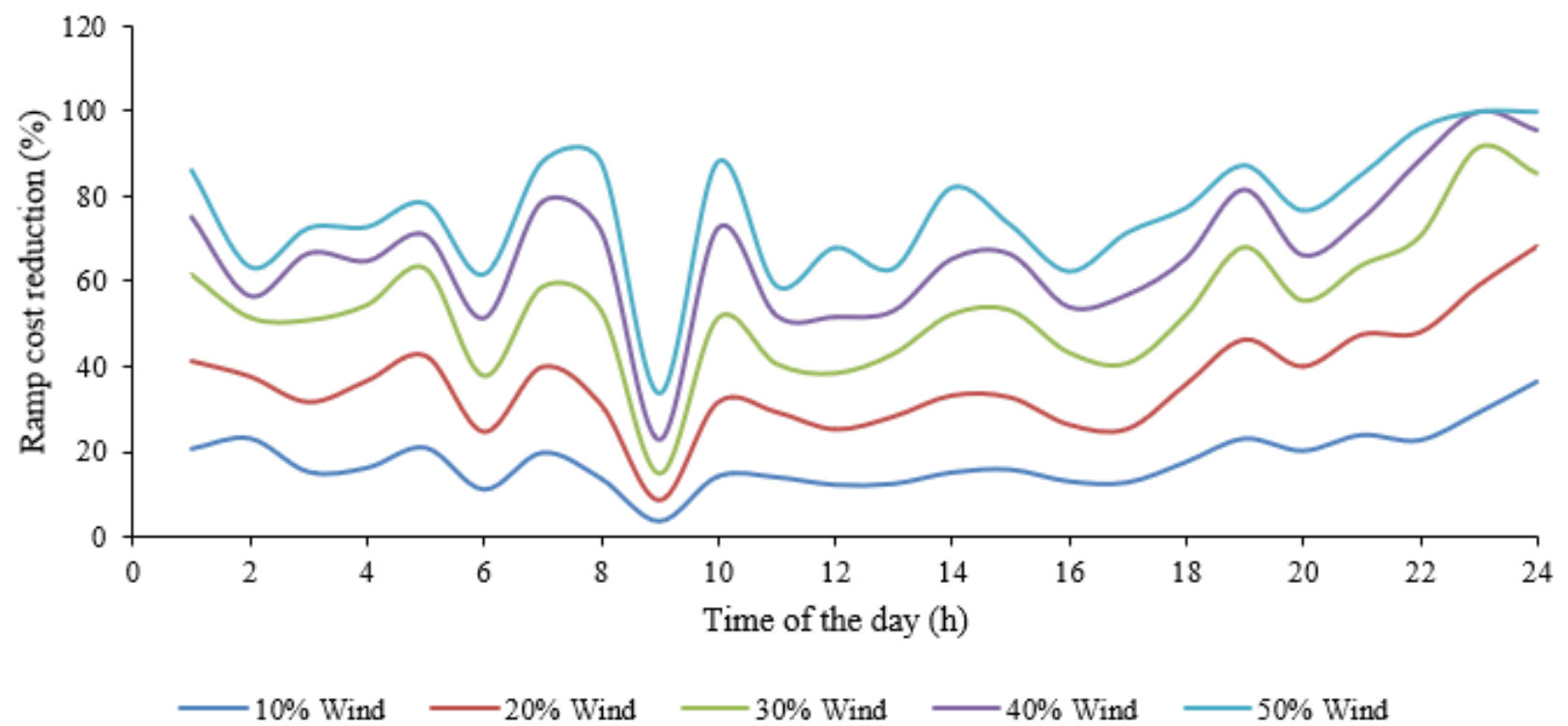

Figure 15. Impact of wind ramping product on system real-time ramp procurement cost at different wind penetration levels

Allowing wind to provide a flexible ramping product proves to yield consistently increasing ramp cost reductions in both the day-ahead and real-time market segments, as shown in Figure 14 and Figure 15. The ramping reserve displacement can also impact generation units' commitment and dispatch. 
Figure 16 shows how the aggregate dispatch of wind and natural gas power plants are affected by the wind ramping product (WRP) in day-ahead, at 40\% wind penetration. Because solar PV plants do not participate in the flexible ramping and their operating cost is low, their output is not affected.

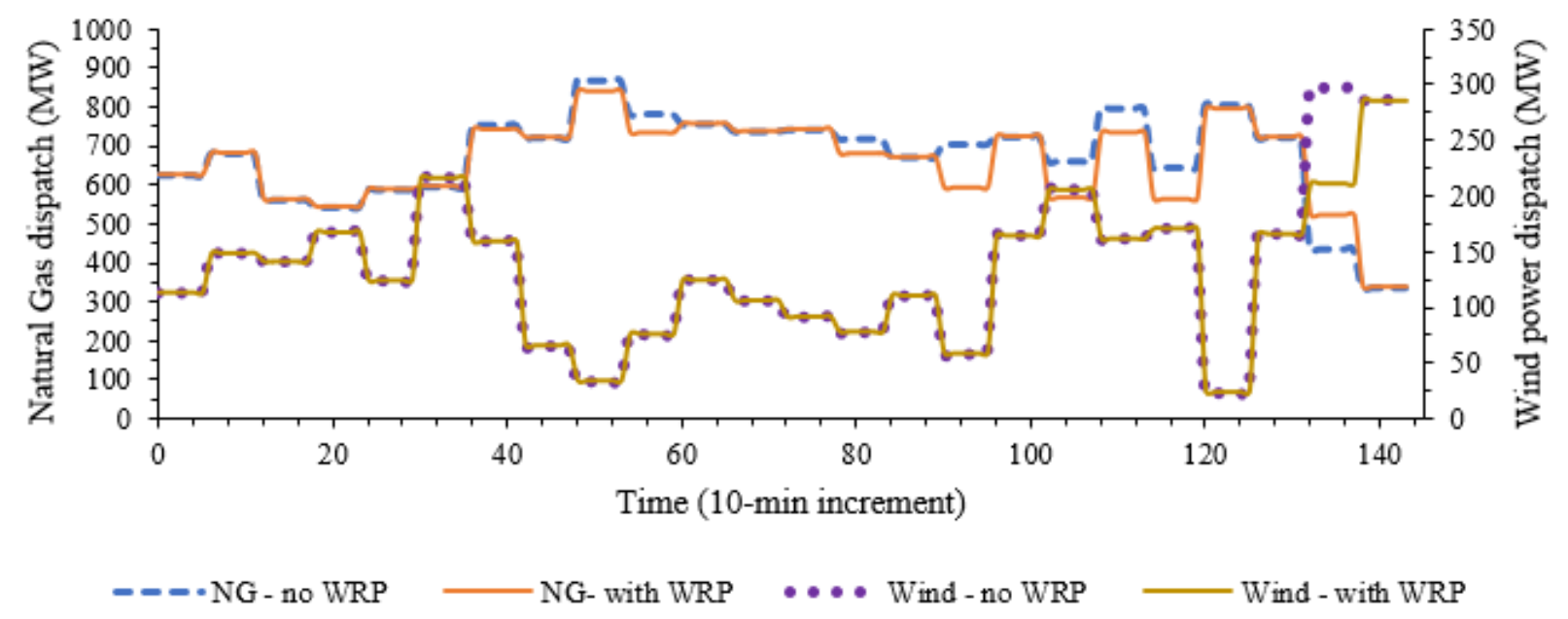

Figure 16. Day-ahead dispatch with and without WRP - $40 \%$ wind penetration

To test the scalability of OpenSMEMS, we perform a wind ramping product impact analysis on the synthetic TAMU 2,000-bus system.

\subsection{Modified TAMU 2,000-Bus System}

The 2000-bus synthetic case is "built from public information and a statistical analysis" on the footprint of the State of Texas. The data is obtained from Electric Grid Test Case Repository (https://electricgrids.engr.tamu.edu/). With 2,000 buses and 3,206 branches, the case boasts a total generation capacity of 96,291.53 MW shared among coal, hydro, natural gas, nuclear, solar, and wind generating units, as presented in Figure 17 and Table 6. The generation fleet is composed of 39 coal power plants, 25 hydroelectric power plants, 4 nuclear power plants, and 367 natural gas power plants, 22 solar PV plants, and 87 wind power plants (WPP). Given that we do not have the water stream time series data, we do not schedule hydro plants the day chosen for our case study. We generate a synthetic normalized time series as a reference shape for solar PV generation. We extract normalized reference wind power output time series from the WIND Toolkit, matching closely station names with WPP bus names. No new WPP was added to the existing ones. Various wind penetration levels are obtained through formulae (3.1). The case studied in this section is the $25 \%$ wind penetration case, i.e., the total wind capacity is one fourth of the total fleet capacity. Figure 17-a shows the generation mix in the original TAMU 2,000 -bus system. The composition of the modified system as considered in this case study is presented in Figure 17-b. The capacity share of all fuel types is shown in Table 6. 
Table 6. Generation capacity shares in the original and the modified TAMU 2000-bus system

\begin{tabular}{lrr}
\hline Fuel Type & Capacity in Original System (MW) & Capacity in Modified System (MW) \\
\hline Coal & $14,501.59$ & $14,501.59$ \\
Hydro & $2,603.37$ & $2,603.37^{*}$ \\
Natural gas & $63,810.22$ & $63,810.22$ \\
Nuclear & $5,138.60$ & $5,138.60$ \\
Solar PV & 650.97 & 650.97 \\
Wind & $9,586.78$ & $28,901.58$ \\
\hline
\end{tabular}

* Not scheduled

We set the ramp cost for each generation unit to $10 \%$ of its marginal production cost given in the TAMU data.

At each bus, we use a nominal load profile weighted by the corresponding demand found in the 2,000-bus power flow case data. For each market stage, we generate the forecast data by superposing the reference weighted demand and a random error signal. Figure 18 shows the aggregate demand profiles considered in different market segments as well the corresponding wind power forecasts.

As in the PJM 5-bus case, the simulation horizon is one day (144 time-slots of 10 minutes each) and processed according the market sequence shown in Figure 10.

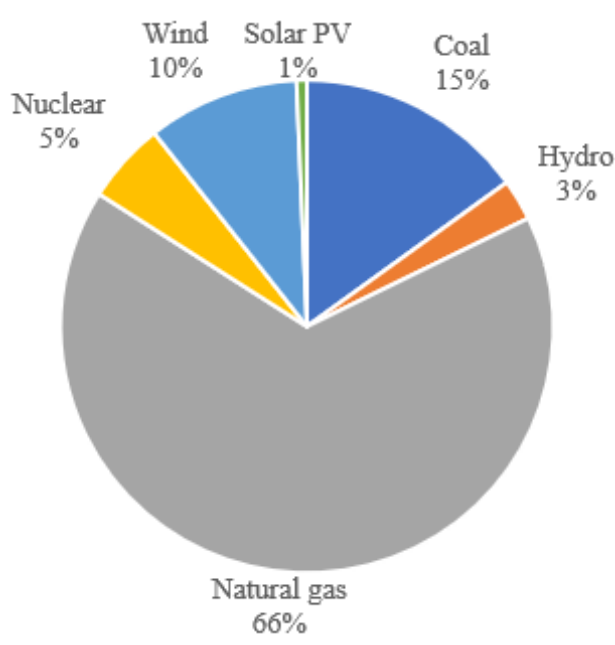

a. Original generation mix

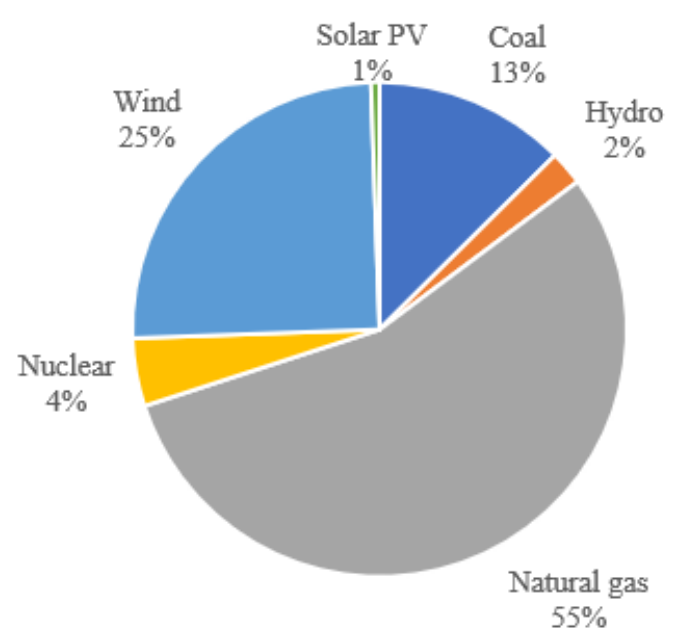

b. Modified generation mix

Figure 17. Generation mix considered in the TAMU 2,000-bus base case 

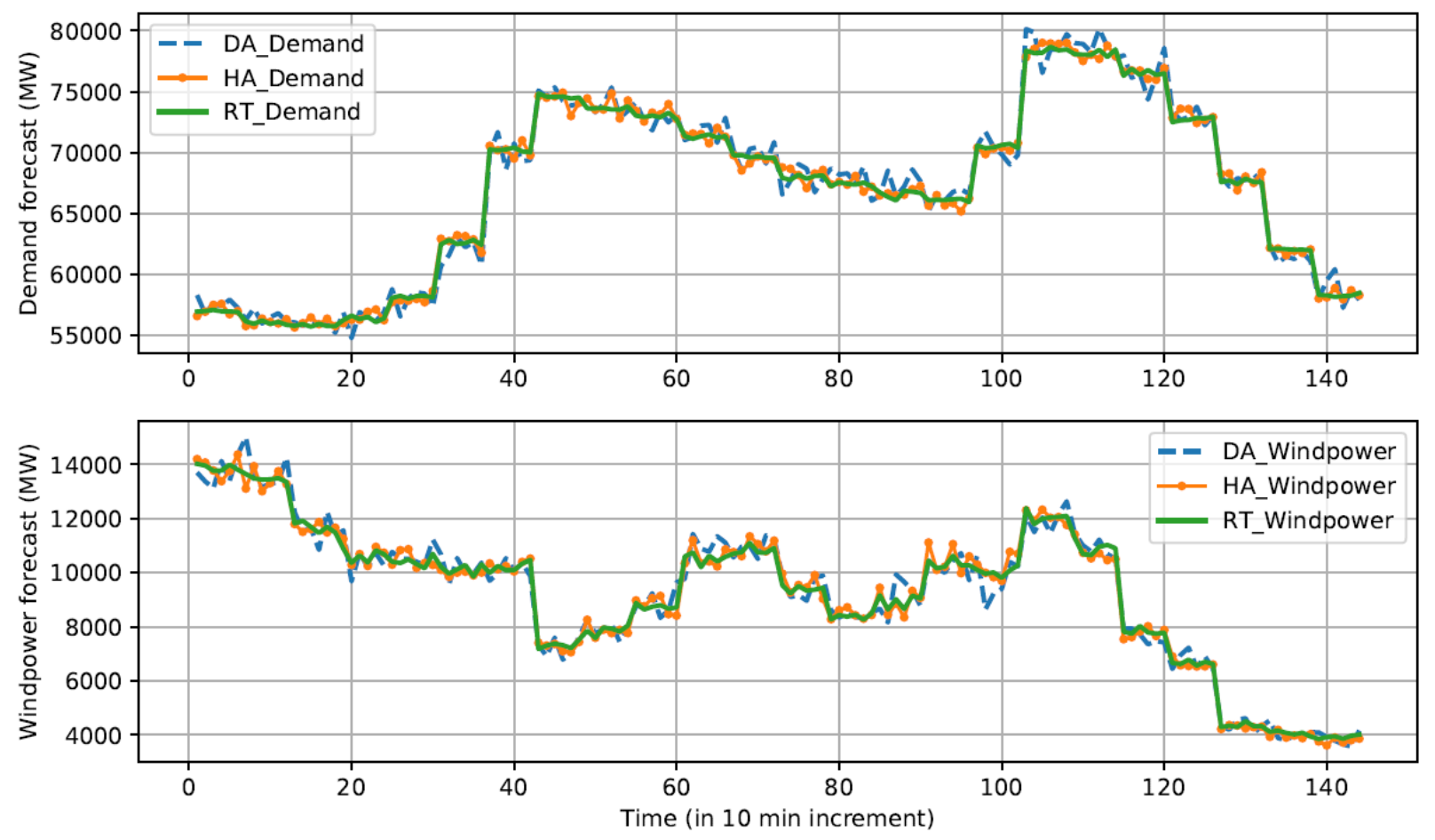

Figure 18. Demand and wind power forecasts in different market segments: TAMU 2,000-bus system

Figures 19 through 22 show OpenSMEMS' dispatch output for the following scenarios:

- No wind plant provides ramping product

- Wind power plants provide ramping product only in day-ahead market

- Wind power plants provide ramping product in real-time (hour-ahead or RT-SCUC stage) only

- Wind power plants offer ramping services in both day-ahead and real-time markets.

Each figure corresponds to the aggregate power dispatch for one fuel type at the time as given by DA-SCUC, RT-SCUC and RT-SCED, respectively. Given that nuclear plants are baseload units, their output is constant throughout all market segments. Thus, their output profiles are not represented. 


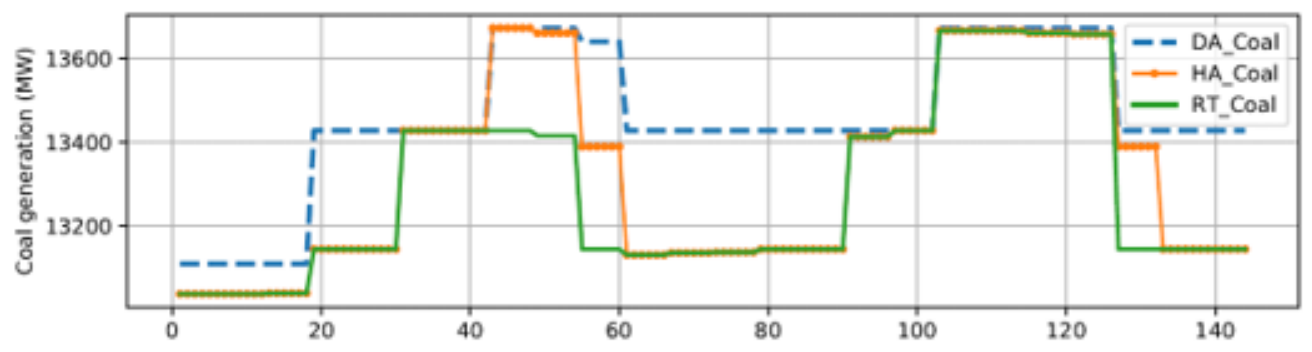

(b) WRP offered in day-ahead only

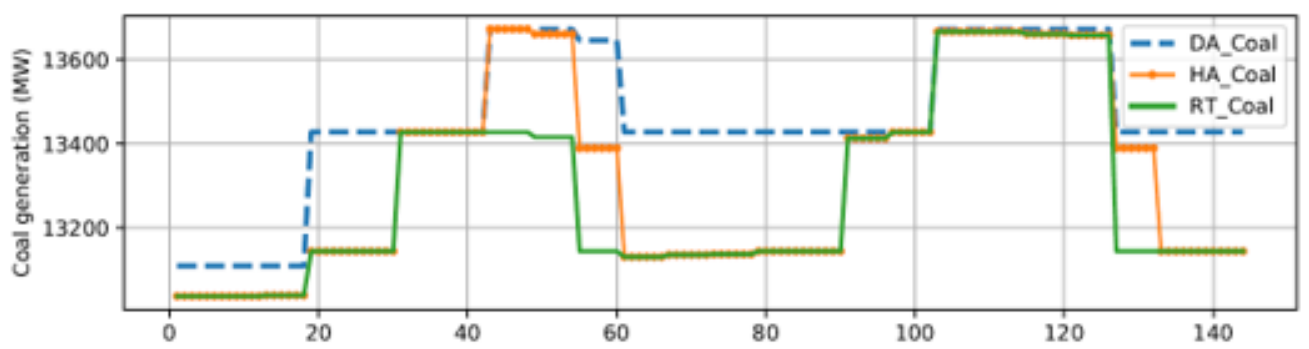

(c) WRP offered in real-time (hour-ahead)

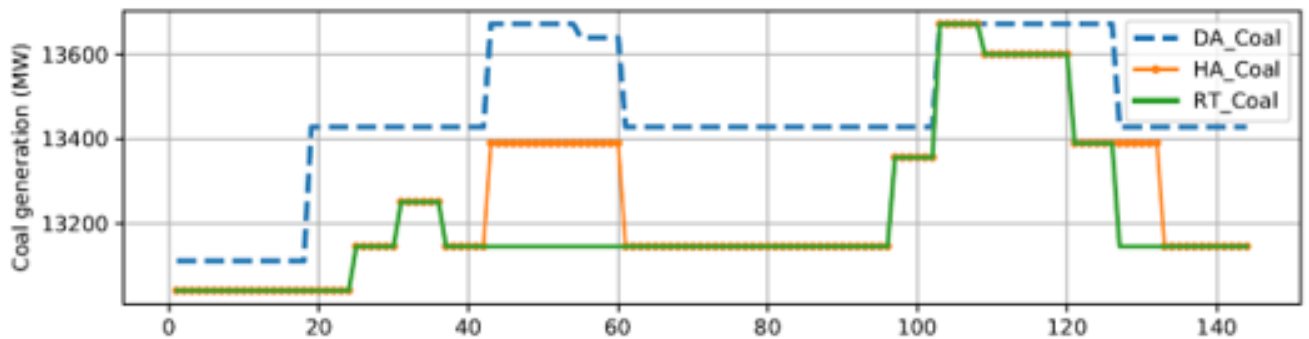

(d) WRP offered in both day-ahead and real-time

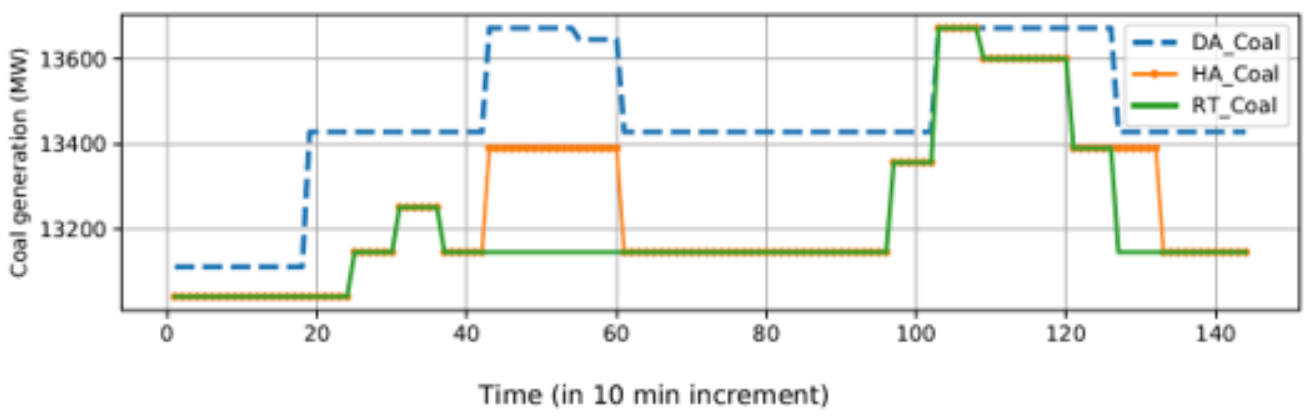

Figure 19. Aggregate coal power output dispatch across all ramping service provision scenarios

As shown in Figure 19, the aggregate dispatch of coal power plants remains consistent through all four WRP provision scenarios. However, the real-time aggregate dispatch is more sensible to whether or not wind provides ramping services, as evidenced by comparing the hour-ahead (HACoal) and real-time (RT-Coal) dispatches in Figure 19-(c) to the ones in Figure 19-(c) for example. 
(a) no WRP

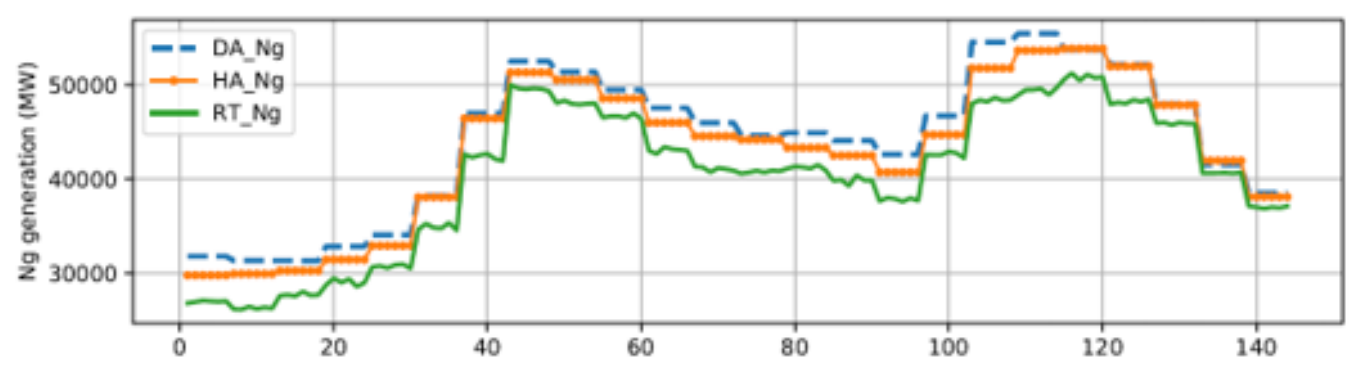

(b) WRP offered in day-ahead only

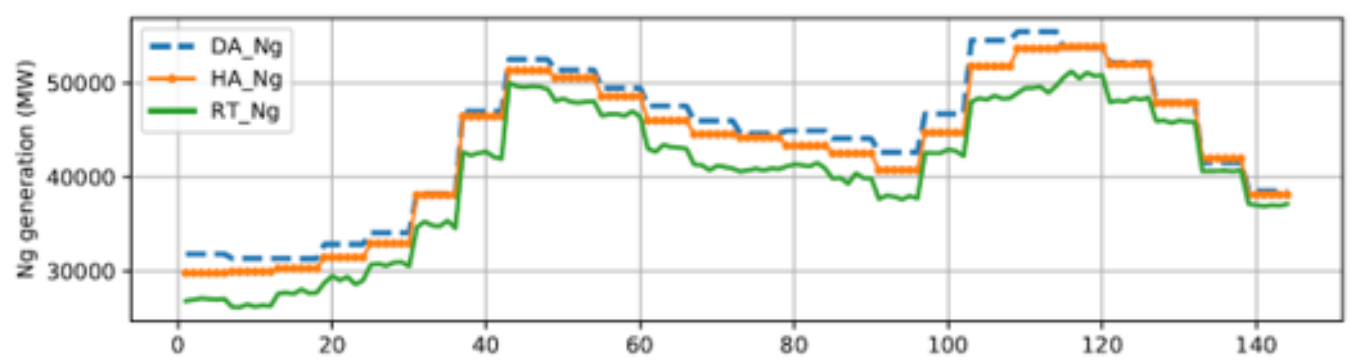

(c) WRP offered in real-time (hour-ahead)

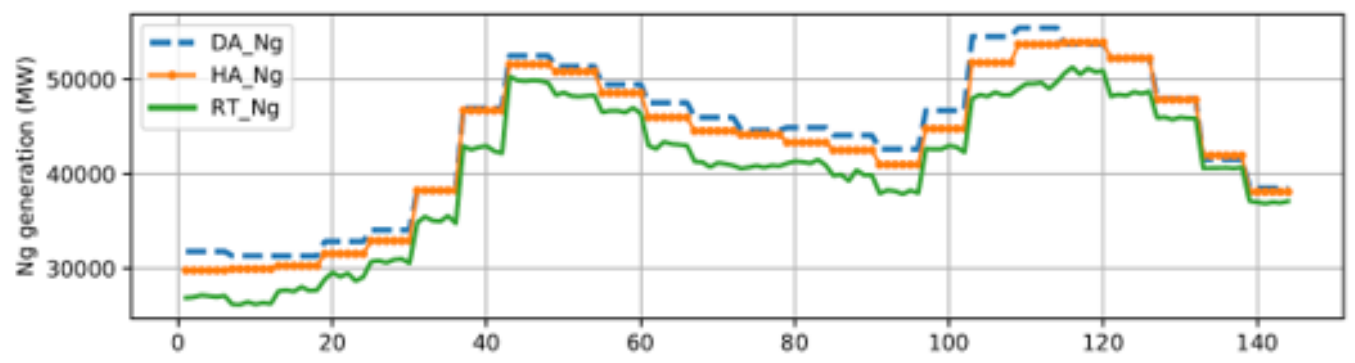

(d) WRP offered in both day-ahead and real-time

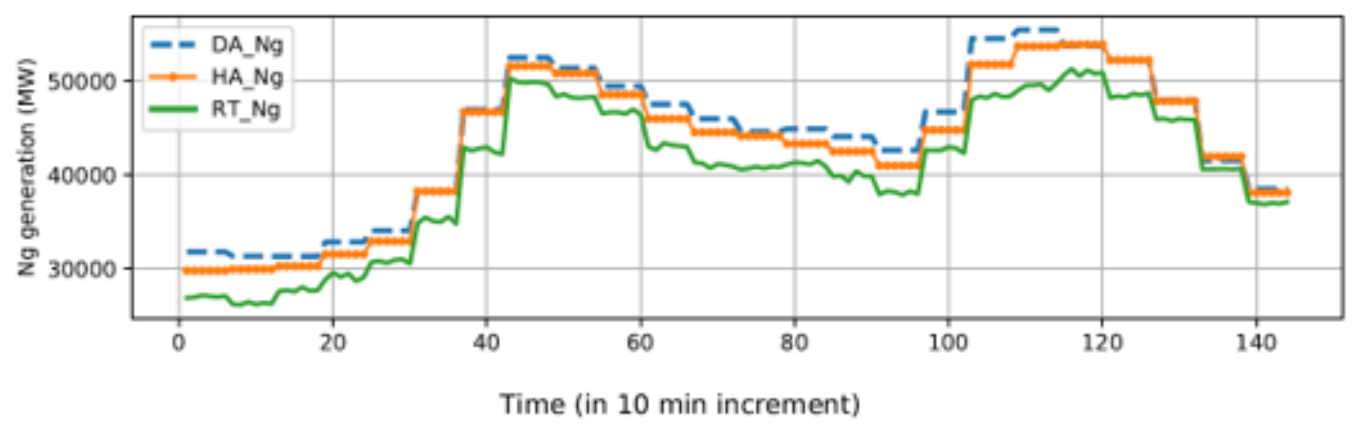

Figure 20. Aggregate natural gas power output dispatch across all ramping service provision scenarios 
(a) no WRP

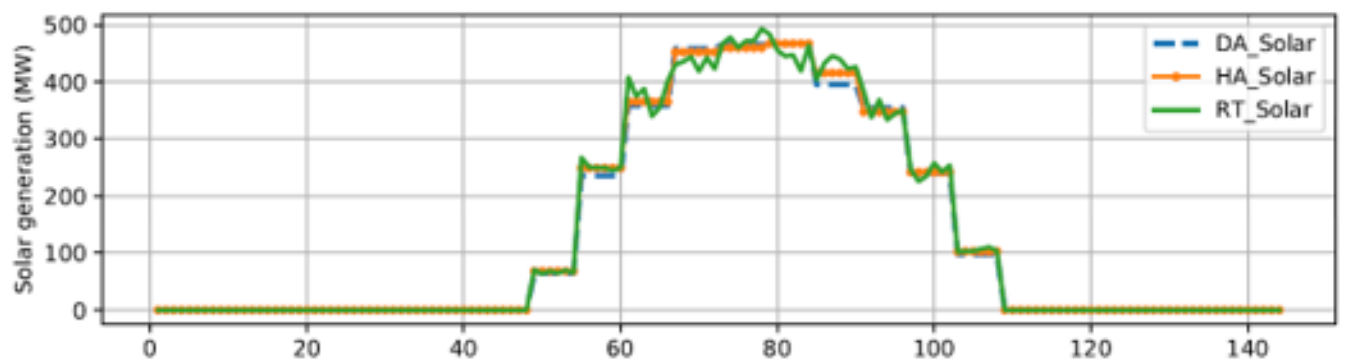

(b) WRP offered in day-ahead only

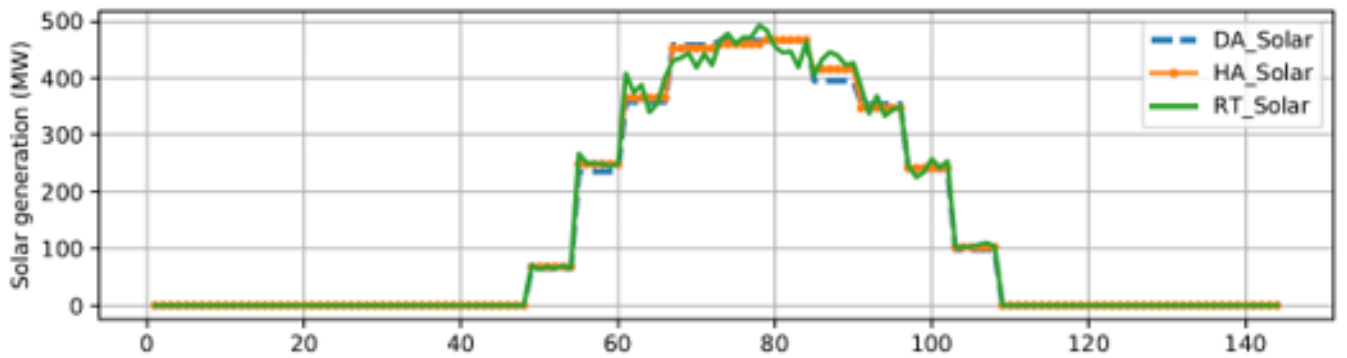

(c) WRP offered in real-time only (hour-ahead)

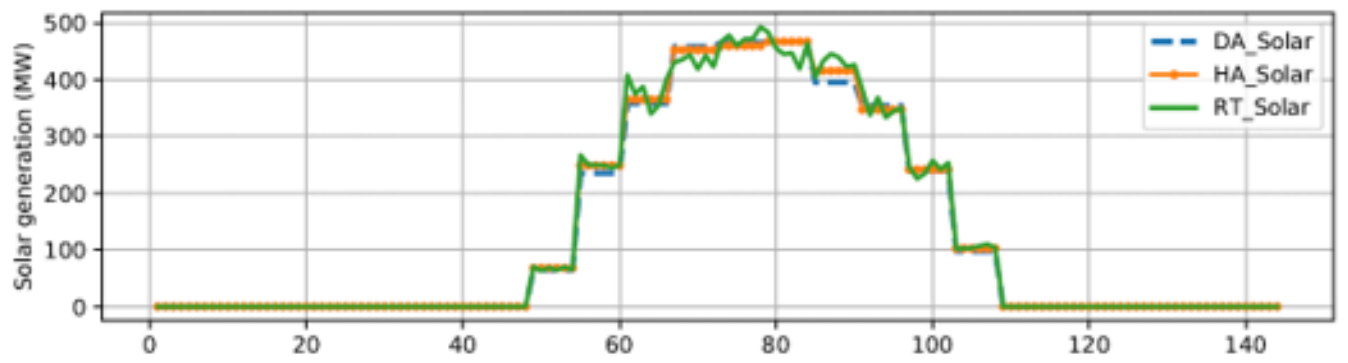

(d) WRP offered in both day-ahead and real-time|

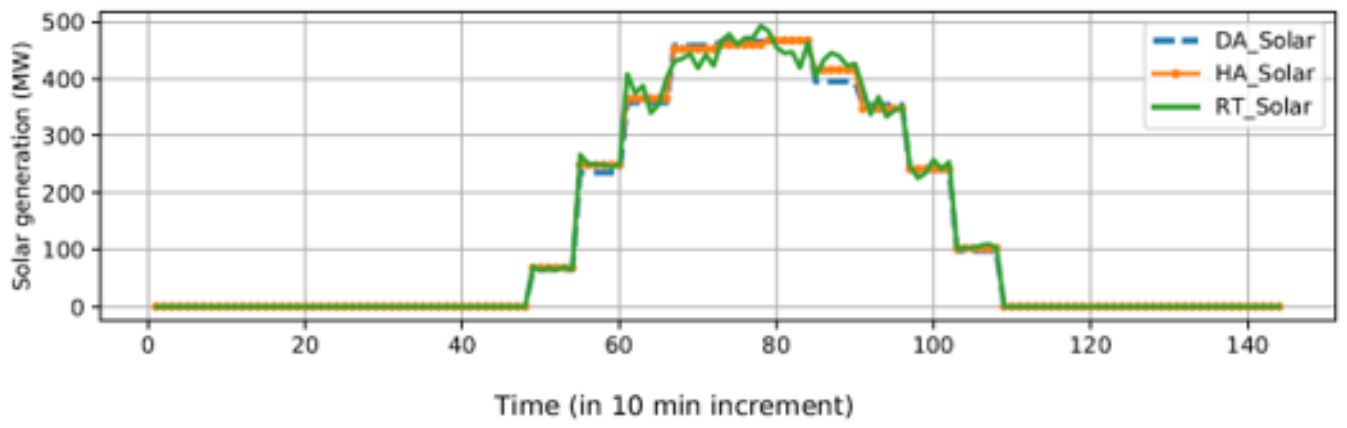

Figure 21. Aggregate solar PV power output dispatch across all ramping service provision scenarios 


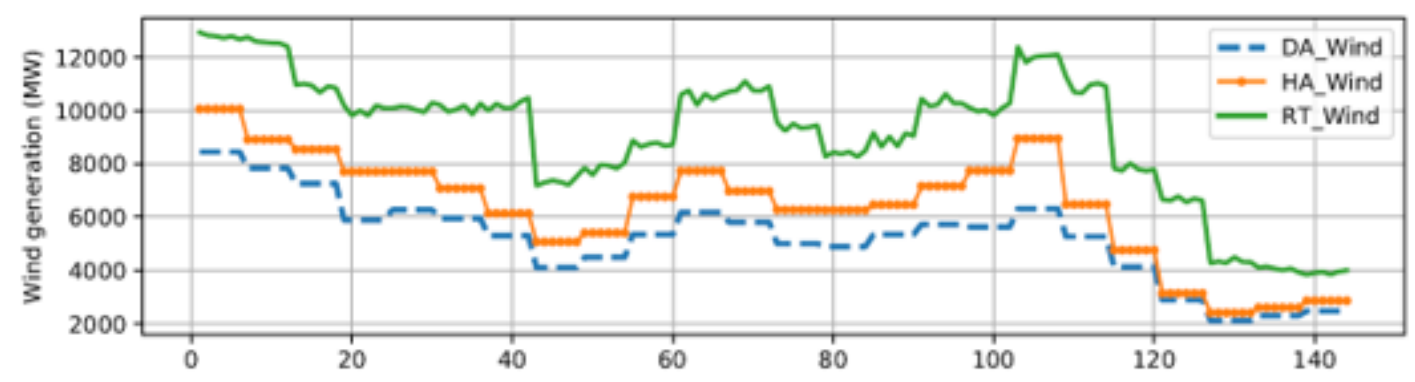

(b) WRP offered in day-ahead only

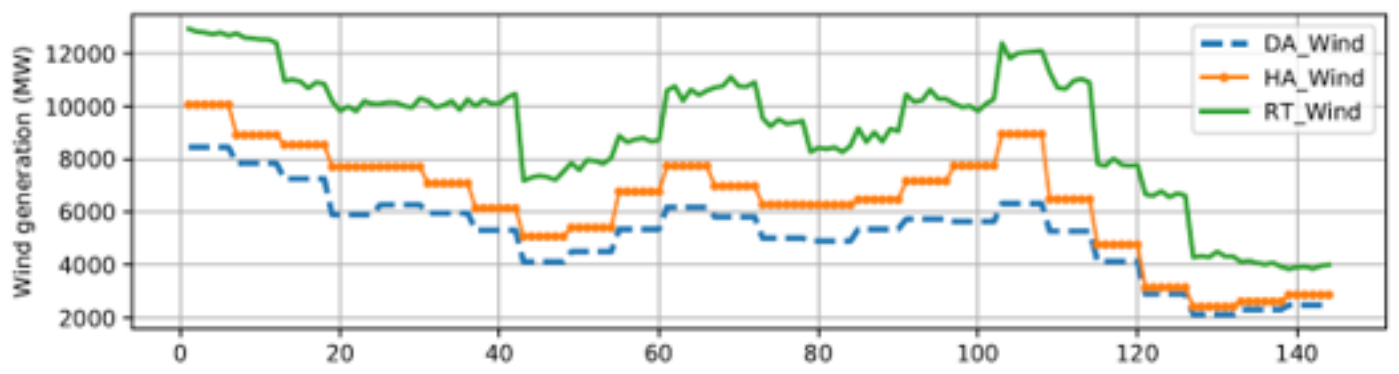

(c) WRP offered in real-time only (hour-ahead)

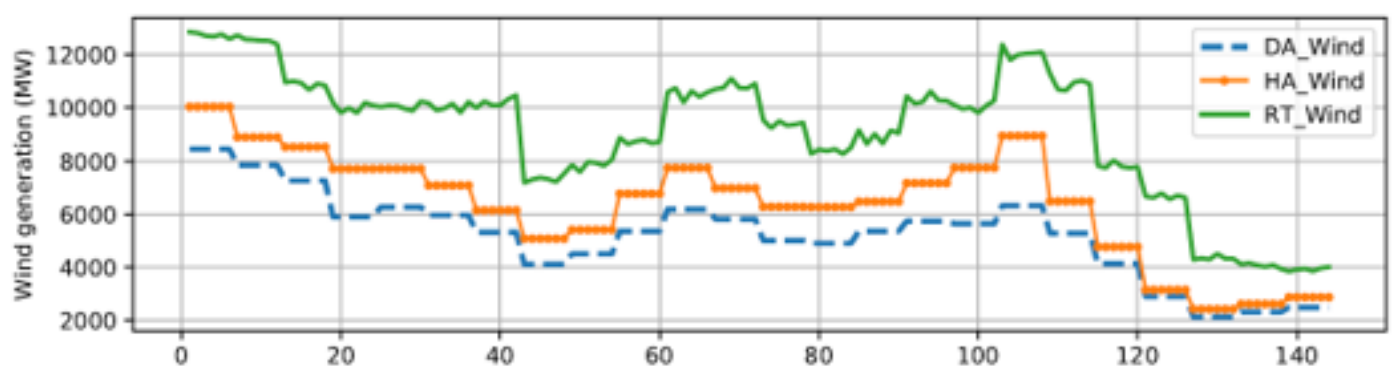

(d) WRP offered in both day-ahead and real-time

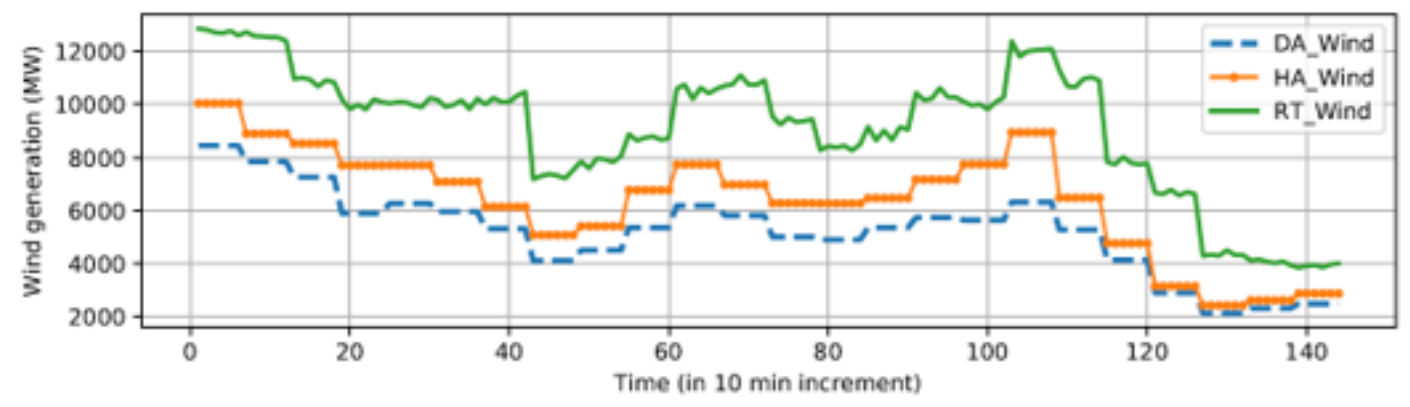

Figure 22. Aggregate wind power output dispatch across all ramping service provision scenarios

The changes in aggregate dispatch for natural gas, solar PV and wind output across WRP scenarios shown in Figures 20, 21 and 22 are not as evident as in the aggregate coal dispatch case presented in Figure 19. We zoom in these changes in Figure 23 for the hour-ahead real-time segment, by means of the dispatch offset defined as the difference between the dispatch with WRP and the dispatch without WRP as given by equation (3.2): 


$$
\text { Dispatch_offset }=\text { Dispatch }_{\text {with } W R P}-\text { Dispatch }_{\text {no WRP }}
$$

As expected, the dispatches affected by WRP scenarios concern the fuel types that participate in the flexible ramping market opportunity, namely, coal, natural gas and wind. Figure 23 shows the hour-ahead dispatch offsets of the aforementioned fuel types. While WRP provision causes a decrease in coal power scheduled output, it leads to and increase in natural gas dispatch. The agregate wind dispatch experiences a slight drop ( 12 MW) in the first 20 time slots. It is worth noting the symmetry of the dispatch offsets, confirming the demand conservation across scenarios with and without WRP.

Without wind providing the flexible ramping products, the flexible natural gas units are operated at lower levels to provide the flexible ramping products. Therefore, the total power outputs from the coal units is higher. However, with wind providing the flexible ramping products, the flexible ramping contributions from the natural gas units reduces. Therefore, the total power output from the natural gas units increases.

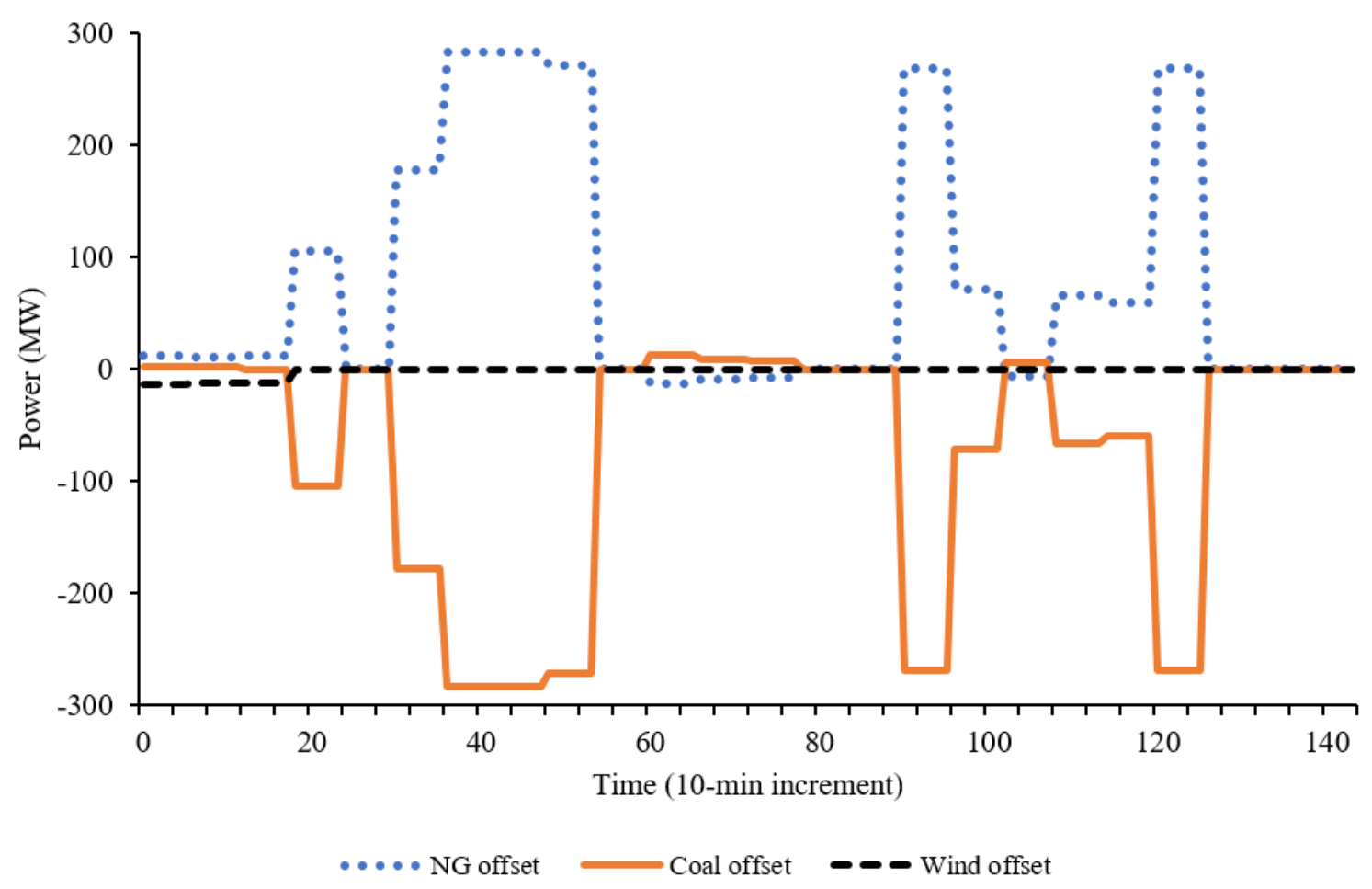

Figure 23. Hour-ahead power dispatch offset

These offsets in the hour-ahead dispatch resulting from adjustments in unit commitments, propagate to the real-time SCED dispatch. This displacement propagation between hour-ahead and RT-SCED is expected because the real-time dispatch inherits from the ahead commitment which updates generation unit availability while considering the latest (and generally, more accurate) demand and variable resource forecasts. Figure 24 shows the real-time aggregate dispatch offsets for natural gas, coal and wind power output. It represents the resulting displacement of real-time economic dispatch, induced by the provision of WRP in the hourahead market segment. The offset trend is similar to the one found in hour-ahead dispatch, as 
anticipated. With wind providing the flexible ramping products, the power output from the natural gas units increases while the output from coal power units decreases. This displacement translates into the reduction of generation-related greenhouse gas emissions, as the most emission-intensive technology shrinks in output in this case. Similar effect can be inferred from Figure 24. In fact, natural gas emits 50 to 60 percent less carbon dioxide (CO2) when combusted in a new, efficient natural gas power plant compared with emissions from a typical new coal plant [NETL 2010]. For reference, analyses have shown that every 10,000 U.S. homes powered with natural gas instead of coal avoids the annual emissions of 1,900 tons of NOx, 3,900 tons of $\mathrm{SO}$, and 5,200 tons of particulates [Alvarez 2012].

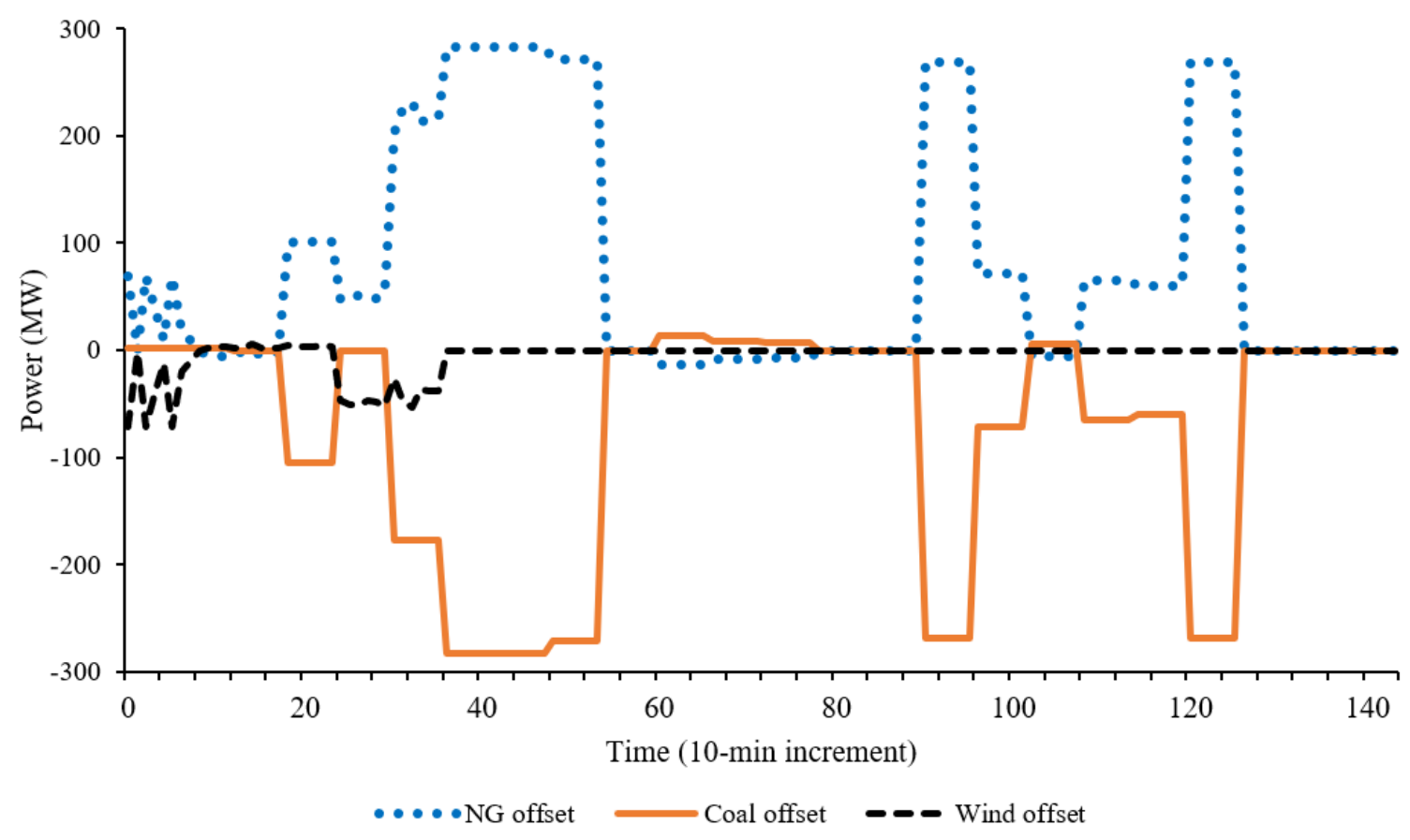

Figure 24. Aggregate real-time power dispatch offset

In addition, the TAMU 2,000-bus test case shows a ramp cost reduction similar to that in the PJM 5-bus case. Table 7 shows the total daily market cost components for all market segments and WRP scenarios, for the TAMU 2,000-bus system case. For reference, the total demand expected in day-ahead is 1,613,596 MWh. The daily demand considered in hour-ahead (RTSCUC) and real-time (RT-SCED) are 1,611,422 MWh and 1,610,373 MWh, respectively. The penalty for load curtailment is $\$ 10,000 / \mathrm{MWh}$.

Before the start of the simulation, all thermal generation units are assumed committed (ON status). In hour-ahead commitment stage (RT-SCUC), because the lookahead horizon is only 2 hours, some units are turned off for economical operation purposes. Hence, the fix cost in hour ahead is higher than in day-ahead where the planning horizon is $24 \mathrm{~h}$, as shown in table 7 . It is also evident that the provision of WRP affects the commitment status of generation units more in hour-ahead than in day-ahead, due to the shorter horizon and frequent status updates in hourahead. In the real time, the available flexible resources are limited compared to the day ahead operation. Consequently, the dispatch of thermal units is also affected. 
The WRP provision results in $72 \%$ ramp cost reduction in day-ahead market and $63 \%$ in realtime market. The overall total cost reduces by $6 \%$ in day-ahead and by $5 \%$ in real-time market. With a total daily demand of 1,613,596 MWh in day-ahead, 1,611,422 MWh in hour-ahead (RTSCUC), and 1,610,373 MWh in real-time (RT-SCED). In this case study, the total daily demand estimate decreases with the time-until-realization. This trend is reflected in the production costs across all market segments. For instance, the production cost in real-time is less than the production cost in hour-ahead. Even though the on/off generation statuses do not change in dayahead with wind providing ramping products, the ramp cost experiences a significance reduction.

In real-time, the provision of wind ramping products impacts the generation statuses as shown by the fix cost column in Table 7 , where the fix cost reduces from $\$ 9,790$ to $\$ 5,975$. This shift in generation schedules is susceptible to affect the emission pattern as mentioned earlier.

Table 7. Sequential daily market cost summary

\begin{tabular}{llrrr}
\hline & WRP Provided in: & Fix Cost $(\$)$ & Ramp Cost $(\$)$ & Production Cost (\$) \\
\hline Day- & None & 0 & $2,096,196$ & $23,350,956$ \\
ahead & Day-ahead & 0 & 589,004 & $23,350,705$ \\
& Hour-ahead & 0 & $2,096,196$ & $23,350,956$ \\
& Day- \& hour-ahead & 0 & 589,004 & $23,350,705$ \\
\cline { 2 - 5 } Hour- & None & 9,790 & $2,098,658$ & $22,544,201$ \\
ahead & Day-ahead & 9,790 & $2,098,658$ & $22,544,201$ \\
& Hour-ahead & 5,975 & 773,235 & $22,545,109$ \\
& Day- \& hour-ahead & 5,975 & 773,235 & $22,545,109$ \\
\cline { 2 - 5 } Real- \\
\cline { 2 - 5 } time & None & - & - & $21,476,930$ \\
& Day-ahead & - & - & $21,476,930$ \\
& Hour-ahead & - & - & $21,478,995$ \\
& Day- \& hour-ahead & - & - & $21,478,995$ \\
\hline
\end{tabular}

Table 8. Simulation time summary of a sequential daily market operation

\begin{tabular}{llr}
\hline Test Case & Market Segment & Time (sec.) \\
\hline PJM 5-bus & Day-ahead & 6.20 \\
& Real-time & 39.60 \\
TAMU 2,000-bus & Day-ahead & $1,900.44$ \\
& Real-time & $14,915.28$ \\
\hline
\end{tabular}

This study reveals that with a proper wind ramping product design and accurate short-term forecasting techniques, wind power plants can significantly participate in providing the muchneeded ramping reserve in an electric power grid with increasing shares of variable generation resources. The examples considered in this report support such a conclusion. The ramp cost decreases consistently with the increase in wind penetration in the PJM 5-bus case for both the 
day-ahead and real-time wind ramping product deployments. Table 7 confirms that the ramp cost when the wind ramping product is provided is always less than the case when it is not. In addition to the ramp cost reduction, the wind ramping product can affect the thermal units' dispatch by deferring their commitments as seen in the TAMU 2,000-bus system case in real time (RT-SCUC). Even in cases where dispatch displacement may not be observed, the wind ramping product displaces the thermal units' ramping reserve. OpenSMEMS is able to capture these details and many more. The total base case simulation time for both the PJM 5-bus system and the TAMU 2,000-bus system are reported in Table 8. It takes approximately 45 seconds to simulate a one-day sequential market operation on the 5-bus system and, 4 hours 40 minutes on the 2000-bus system. The differences in simulation times for each WRP scenario compared to the base case scenario, are negligible. The simulations were performed on a computer with the following characteristics: Processor Intel (R) Core i5-7300U CPU@2.60 GHz, 8 GB RAM and 64-bit OS. 


\section{Conclusion}

In this project, an innovative, integrated, and transformative approach to mitigate the impact of wind ramping is proposed. Through probabilistic wind ramp forecasting, wind power has the capability to provide flexible ramping products. By making wind power dispatchable and allowing the efficient management of wind ramping characteristics, wind integration costs can be significantly reduced.

A Python based open source, sequential, multi-timescale electricity market simulation tool (OpenSMEMS) is developed in this project. The day-ahead SCUC, real-time SCUC, and realtime SCED are sequentially simulated with updated load and renewable forecasting information. Then the wind flexible ramping products are investigated at different timescales in the electricity market simulation, including both the day-ahead and real-time operations. From both the small 5-bus system and 2,000-bus case studies, it is demonstrated that the system operating cost and the ramping cost can be reduced with wind power providing the flexible ramping products. Whether the wind flexible ramping products are provided in the day-ahead or real-time operation, the system operating cost and the ramping cost are reduced. When wind power provides flexible ramping products in the day-ahead market, the reduction in the system operating cost is larger than the case with wind providing flexible ramping products in the realtime market. When provided in the day-ahead, wind ramping products result in a $72 \%$ ramp cost reduction and a $6 \%$ total system cost reduction, and the real-time market wind ramping product provision leads to a $63 \%$ ramp cost reduction and a $5 \%$ total system cost reduction for the 2,000bus system considered. Therefore, per the case studies conducted in this project, wind providing flexible ramping has a higher cost impact in the day-ahead market than in real time; however, the impact on generation schedules proves to be higher in real time than in the day-ahead. In addition to reducing system operating costs, wind ramping products induce fuel-type generation displacement that can translate into emissions reduction based on the flexibility of the different technologies at hand. In fact, in the cases studied, when wind power plants provide ramping services, the energy generation of coal power plants is displaced by that of natural gas power plants. In such a case, a fraction of generation-related greenhouse gas emission is avoided by allowing wind to provide ramping services. 


\section{References}

California Independent System Operator (CAISO). 2012. Flexible Ramping Products. Folsom, CA: April 9, 2012. https://www.caiso.com/Documents/DraftFinalProposal-

FlexibleRampingProduct.pdf.

Pacific Gas and Electric Company (PG\&E). 2019. "Pacific Gas and Electric's Comments on Flexible Ramping Product Refinements Issue Paper/Straw Proposal.” November 14, 2019. http://www.caiso.com/InitiativeDocuments/PGECommentsFlexibleRampingProductRefinements-IssuePaper-StrawProposal.pdf .

Midcontinent Independent System Operator (MISO). 2013. "Ramp Product Conceptual Design Whitepaper." July 10, 2013. https://cdn.misoenergy.org/Ramp\%20Capability\%20for\%20Load\%20Following\%20in\%20MIS O\%20Markets\%20White\%20Paper271169.pdf.

Bodini, Nicola, Dino Zardi, and Julie K. Lundquist. 2017. "Three-Dimensional Structure of Wind Turbine Wakes as Measured by Scanning Lidar." Atmospheric Measurement Techniques 10: 2881-2896. https://doi.org/10.5194/amt-10-2881-2017.

Draxl, Caroline, Bri-Mathias Hodge, Andrew Clifton, and Jim McCaa. 2015. Overview and Meteorological Validation of the Wind Integration National Dataset Toolkit (NREL/TP-500061740). Golden, CO: National Renewable Energy Laboratory. https://www.nrel.gov/docs/fy15osti/61740.pdf.

Cui, Mingjian, Jie Zhang, Hongyu Wu, and Bri-Mathias Hodge. 2017. "Wind-Friendly Flexible Ramping Product Design in Multi-Timescale Power System Operations." IEEE Transactions on Sustainable Energy 8, no. 3: 1064-1075. 10.1109/TSTE.2017.2647781.

Cui, Mingjian, Qin Wang, Venkat Krishnan, Bri-Mathias Hodge, and Jie Zhang. 2019. "A DataDriven Methodology for Probabilistic Wind Power Ramp Forecasting." IEEE Transactions on Smart Grid 10, no. 2: 1326-1338. 10.1109/TSG.2017.2763827.

Cui, Mingjian, Venkat Krishnan, Bri-Mathias Hodge, and Jie Zhang. 2019. "A Copula-Based Conditional Probabilistic Forecast Model for Wind Power Ramps." IEEE Transactions on Smart Grid 99, no. 99. 10.1109/TSG.2018.2841932.

Fang, Xin, Bri-Mathias Hodge, and Venkat Krishnan. 2018. "Strategic Offering for Wind Power Producers Considering Both Energy and Flexible Ramp Products.” Energies 11, no. 5. https://doi.org/10.3390/en11051239.

Fang, Xin, Bri-Mathias Hodge, Ershun Du, Chongqing Kang, and Fangxing Fran Li. 2019. "Introducing Uncertainty Components in Locational Marginal Prices for Pricing Wind Power and Load Uncertainties." IEEE Transactions on Power Systems 34, no. 3. 10.1109/TPWRS.2018.2881131. 
Fang, Xin, Bri-Mathias Hodge, Ershun Du, Ning Zhang, and Fangxing Li. 2018. "Modelling Wind Power Spatial-Temporal Correlation in Multi-Interval Optimal Power Flow: A Sparse Correlation Matrix Approach." Applied Energy 230: 531-539.

https://doi.org/10.1016/j.apenergy.2018.08.123.

Fang, Xin, Bri-Mathias Hodge, Venkat Krishnan, and Fangxing Li. 2018. "Potential of Wind Power to Provide Flexible Ramping Products and Operating Reserve." In Proceedings of the IEEE PES General Meeting 2018, Portland, Oregon, August 16-20, 2018.

10.1109/PESGM.2018.8586436.

Huang, Bing, Venkat Krishnan, and Bri-Mathias Hodge. 2018. "Analyzing the Impacts of Variable Renewable Resources on California Net-Load Ramp Events." In Proceedings of the IEEE PES General Meeting, Portland, Oregon, August 16-20, 2018.

https://www.osti.gov/servlets/purl/1465933.

Sedzro, Kwami, Xin Fang, and Bri-Mathias Hodge. 2018. "Analysis of Wind Ramping Product Formulations in a Ramp-constrained Power Grid." Presented at the Hawaii International Conference on System Science, January 8-11, 2018. http://hdl.handle.net/10125/59777.

Fang, Xin, Sedzro, Kwami, Yuan, Haoyu, Ye, Hongxing, Hodge, Bri-Mathias. "Deliverable Flexible Ramping Products Considering Spatiotemporal Correlation of Wind Generation and Demand Uncertainties.” IEEE Transactions on Power Systems 99, no. 99.

10.1109/TPWRS.2019.2958531.

Zhu, Lei, Jacob Holden, Eric Wood, and Jeffrey Gender. 2017. "Green Routing Fuel Saving Opportunity Assessment: A Case Study Using Large-Scale Real-World Travel Data.” Presented at the 2017 IEEE Intelligent Vehicles Symposium (IV), Los Angeles, California, June 11-14, 2017. https://doi.org/10.1109/IVS.2017.7995882.

National Energy Technology Laboratory (NETL). 2015. Cost and Performance Baseline for Fossil Energy Plants, Volume 1: Bituminous Coal and Natural Gas to Electricity: Revision 2. (DOE/NETL-2015/1723). Washington, D.C.: U.S. Department of Energy. https://www.netl.doe.gov/projects/files/CostandPerformanceBaselineforFossilEnergyPlantsVolu melaBitCoalPCandNaturalGastoElectRev3 070615.pdf

Alvarez, Ramón A., Stephen W. Pacala, James J. Winebrake, William. L. Chameides, and Steven P. Hamburg. 2012. "Greater Focus Needed on Methane Leakage from Natural Gas Infrastructure." Proceedings of the National Academy of Sciences 109: 6435-6440. https://doi.org/10.1073/pnas.1202407109. 